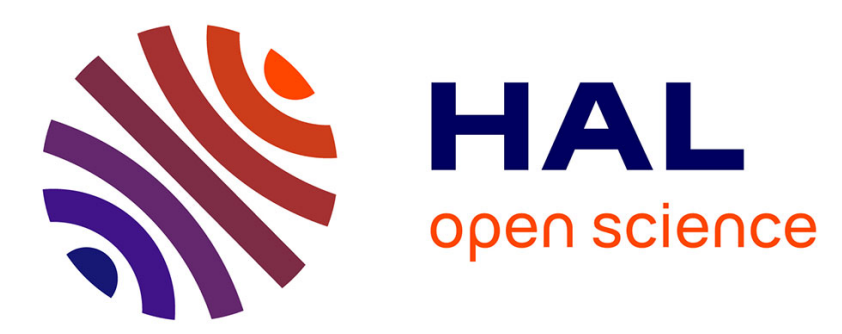

\title{
On the construction of discrete directional wavelets: HDWT, space-frequency localization and redundancy factor \\ Sylvain Durand
}

\section{- To cite this version:}

Sylvain Durand. On the construction of discrete directional wavelets: HDWT, space-frequency localization and redundancy factor. Multiscale Modeling and Simulation: A SIAM Interdisciplinary Journal, 2009, 7 (3), pp.1325-1347. hal-00430224

\section{HAL Id: hal-00430224 \\ https://hal.science/hal-00430224}

Submitted on 6 Nov 2009

HAL is a multi-disciplinary open access archive for the deposit and dissemination of scientific research documents, whether they are published or not. The documents may come from teaching and research institutions in France or abroad, or from public or private research centers.
L'archive ouverte pluridisciplinaire HAL, est destinée au dépôt et à la diffusion de documents scientifiques de niveau recherche, publiés ou non, émanant des établissements d'enseignement et de recherche français ou étrangers, des laboratoires publics ou privés. 


\title{
On the construction of discrete directional wavelets: HDWT, space-frequency localization and redundancy factor
}

\author{
Sylvain Durand \\ MAP5, CNRS UMR 8145, 45 rue des Saints Pères, 75270 Paris Cedex 06, France \\ e-mail: sylvain.durand@mi.parisdescartes.fr
}

\begin{abstract}
We address the issue of constructing directional wavelet bases. After considering orthonormal directional wavelets whose Fourier transforms are indicator functions, we give a construction of directional wavelets with fast decay that is based on an hexagonal filter bank tree. An implementation for squarely sampled images and numerical results are presented. Then we discuss the frequency localization of directional wavelet bases. We analyze the incompatibility between a proper localization and the non-redundancy constraint, and show that the non permissibility condition can be extended to general wavelet bases that are not necessary generated by a filter bank tree. At last, we show that there exist directional wavelet tight frames that are well localized and have a redundancy factor arbitrary close to 1 .
\end{abstract}

Key words. Wavelet transform, directional wavelets, two-dimensional filter banks. AMS Subject Classifications. 42C40, 65T60, 68U10, 94A08.

\section{Introduction}

Because of their ability to characterize piecewise smooth functions with few coefficients, wavelets have been a successful tool in several signal and image processing tasks. Wavelets for $2 \mathrm{D}$ images are typically constructed by tensor products of $1 \mathrm{D}$ wavelets. However the obtained separable wavelets do not provide sparse representations of every image's structures. Indeed a common image model is based on the observation that if one zooms in on a detail of an ordinary image, its geometrical flow is generally, nearly uniform. In other words, its local Fourier transform is concentrated along a line which is orthogonal to the flow (see Fig. 1). When the image is decomposed in a separable wavelet basis, its energy is spread on many coefficients (see Fig. 2-(a)). Moreover, both diagonal directions are ambiguously represented by a single wavelet. Although the wavelet transform provides sparse (but not optimal) representations of horizontal and vertical singularities (using the horizontal and the vertical wavelets), it does not permit to represent efficiently singularities which are positioned along other directions.

For more than a decade, new transforms have been proposed in order to make up for this lack of directionality of separable wavelets. Several adaptative transforms were developed. For instance, first generation bandelets[17] consist in warping separable 


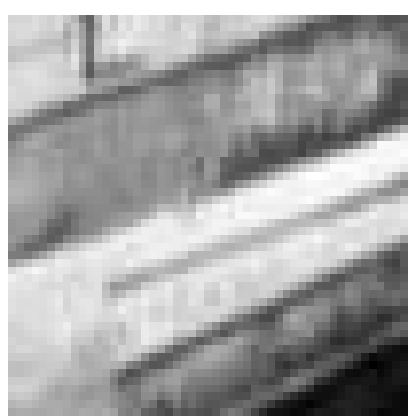

(a)

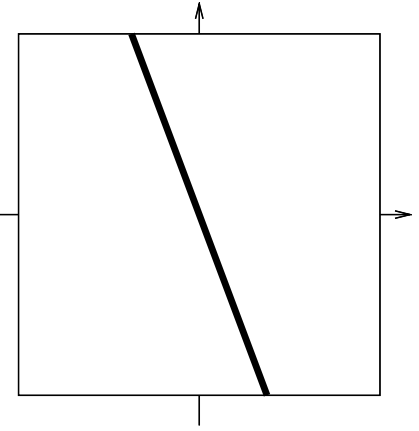

(b)

Figure 1: (a) Detail of an image. (b) Its spectrum is concentrated along a line.

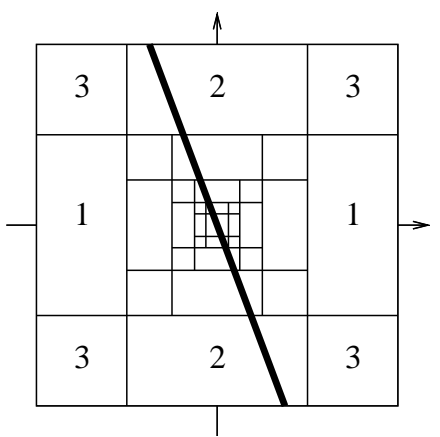

(a)

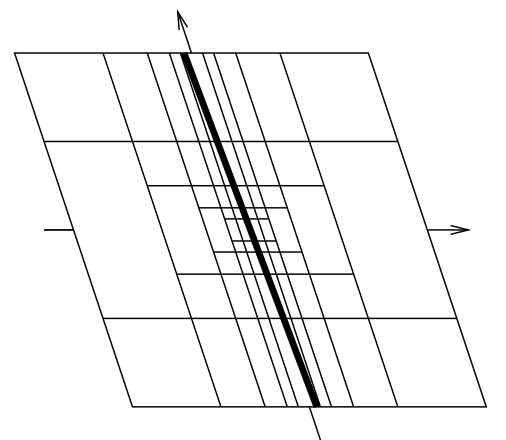

(b)

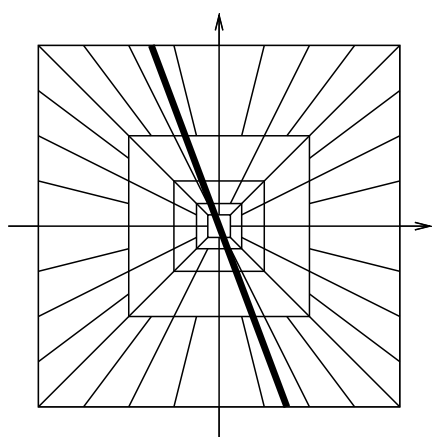

(c)

Figure 2: Frequency support of (a) Wavelets, (b) Bandelets (first generation), (c) Curvelets. 
wavelets along the geometrical flow of images, and performing a bandeletization (see Fig. 2-(b)). We focus in this article on the construction of non adaptative transforms that are generally implemented in a faster algorithm as they do not require prior information on the image. Among the most prominent non-adaptative, directional transforms, one may mention hexagonal wavelets [24, 9], directional filter banks [2], steerable pyramids [25], directional wavelets [21, 1], brushlets [20], dual-tree complex wavelets [16, 23], ridgelets [4], curvelets [5] (see Fig. 2-(c)) and contourlets [11]. All these transforms are of great interest for applications in fields such as compression, denoising, enhancement and oriented feature extraction. Their construction leaves however some open questions. Indeed, in compression, powerful and practical transforms must satisfy the following requirements

- Arbitrary fine directions

- Good spatial localization

- Good frequency localization

- Fast and accurate implementation

- No redundancy.

But none of the quoted above transforms satisfies all these properties. The last one is crucial in the approach we adopted. Curvelets and contourlets are known to perform very well at low bit rate compression. But they are redundant frames. As a consequences, separable wavelets remain sometimes more efficient to compress images at rates that are used in practice. Let us emphasize that, in this article, we are thinking of compression applications. If one deals with denoising, for instance, the constraint "no redundancy" has to be replaced by "shift invariance".

Directional wavelets were introduced in [21]. A family of wavelets is generated from a single function, called mother wavelet, by translation, dilation and rotation,

$$
a \psi\left(a R_{\theta} \cdot-b\right), \quad a \in \mathbb{R}_{+}, b \in \mathbb{R}^{2}, R_{\theta} \in S O(2, \mathbb{R}) .
$$

The 3 parameters $a, b, \theta$ can be discretized in order to obtain tight frames for $L^{2}\left(\mathbb{R}^{2}\right)$. Curvelets are constructed with a more subtle family of rotation-dilation operators. Indeed it is proved in [5] that one obtains sparser representations of some classes of images by using functions that have a finer angular resolution at finer scales, following a parabolic scaling law. (At scale $a$ the angular resolution must be $O(\sqrt{a})$.) For that reason, we consider the more general family (that we still call directional wavelets)

$$
\left\{\left|\operatorname{det} D_{k}\right| \psi\left(D_{k} \cdot-\gamma\right)\right\}_{k \in \mathcal{K}, \gamma \in \Gamma}=\left\{\psi_{k}(\cdot-\gamma)\right\}_{k \in \mathcal{K}, \gamma \in \Gamma_{k}}
$$

where $\Gamma$ is a regular lattice on $\mathbb{R}^{2}$ and, for all $k, \psi_{k}=\left|\operatorname{det} D_{k}\right|^{1 / 2} \psi\left(D_{k} \cdot\right), \Gamma_{k}=$ $D_{k}^{-1} \Gamma$, and $D_{k}$ is a linear operator on $\mathbb{R}^{2}$. The sequence $\left\{D_{k}\right\}_{k \in \mathcal{K}}$ can be constructed following the theory of wavelets with composite dilations [14] although more general sequences are considered in this article. In order to complete the basis, one has to consider also a low frequency function $\phi$ that we can denote $\psi_{0}$ but that it is not obtained by dilating the mother wavelet $\psi$. At last, in order to obtain bases for $L^{2}\left(\mathbb{R}^{2}\right)$, we will not always assume that $\psi_{k}=\left|\operatorname{det} D_{k}\right|^{1 / 2} \psi\left(D_{k} \cdot\right)$ but that $\psi_{k}$ is close to $\left|\operatorname{det} D_{k}\right|^{1 / 2} \psi\left(D_{k} \cdot\right)$ in a sense that will be specified below. Notice that the same kind of hypothesis is made to obtain second generation curvelets tight frames [3]. 
In Section 2, the construction of directional wavelet bases is restricted to wavelets that are Fourier transforms of indicator functions. In order to meet the above requirements, these wavelets have to be approximated by functions with fast decay. In that direction, we presents a numerical implementation of one of the transforms introduced in [13]. It is implemented in a fast algorithm that is based on a filter bank tree with hexagonal and 2-channels filter banks. This hexagonal directional wavelet transform is defined in Section 3.1. In Section 3.2, we propose an implementation for squarely sampled images, while in Section 3.3, we give some numerical results and comparisons. In Section 4, we consider the frequency localization of directional wavelets with fast decay. While it satisfies all the other, cited above, required properties, the proposed hexagonal directional wavelet transform fails to achieve a perfect frequency localization. General wavelets that are not necessary generated by filter bank trees are considered in Section 4.2. The incompatibilities between all the required properties is addressed in a statement that generalizes the permissibility condition for filter banks. In order to circumvent this obstruction in the construction of directional wavelet bases, we show, in Section 5, that there are directional wavelet tight frames that are well localized in the space and in the frequency domains, and that have arbitrary small redundancy factors. At last, a conclusion is drawn in Section 6.

\section{Shannon Directional Wavelet Bases}

As it is shown in Figure 2, the issue of obtaining sparse representations of images can be addressed by constructing tilings of the frequency domain. In this section, the frequency localization is therefore singled out as one consider wavelets whose Fourier transforms are indicator functions. The next sections are devoted to the issue of approximating these basis functions with wavelets which are well localized in space and frequency domains.

Assume the Fourier transform of $\psi_{k}^{s}$ is

$$
\widehat{\psi_{k}^{s}}=2 \pi\left|A_{k}\right|^{-1 / 2} \chi_{A_{k}}
$$

where $A_{k}$ is an open subset of $\mathbb{R}^{2},\left|A_{k}\right|$ is its area and $\chi_{A_{k}}$ is the indicator function of $A_{k}$. Recall that the dual lattice $\Gamma^{*}$ of a lattice $\Gamma \subset \mathbb{R}^{2}$ is defined by

$$
\Gamma^{*}=\left\{\gamma \in \mathbb{R}^{n}:\langle\eta, \gamma\rangle \in 2 \pi \mathbb{Z}, \forall \eta \in \Gamma\right\}
$$

so that the Fourier transform is an isomorphism between $\ell^{2}(\Gamma)$ and $L^{2}\left(\mathbb{R}^{2} / \Gamma^{*}\right)$. (See the appendix for more informations on the Fourier transform on regular lattice.)

Proposition 1 The family $\left\{\psi_{k}^{s}(\cdot-\gamma)\right\}_{k \in \mathcal{K}, \gamma \in \Gamma_{k}}$ is an orthonormal basis for $L^{2}\left(\mathbb{R}^{2}\right)$ if and only if

1. $\left\{A_{k}+\{\gamma\}\right\}_{\gamma \in \Gamma_{k}^{*}}$ is a tiling of $\mathbb{R}^{2}$,

2. $\left\{A_{k}\right\}_{k \in \mathcal{K}}$ is a tiling of $\mathbb{R}^{2}$.

Proof. The main idea of the proof of this proposition is that Condition 1 is satisfied if and only if $\left\{\psi_{k}^{s}(\cdot-\gamma)\right\}_{\gamma \in \Gamma_{k}}$ is an orthonormal basis for

$$
\mathcal{W}_{k}=\left\{f \in L^{2}\left(\mathbb{R}^{2}\right): \operatorname{supp} \hat{f} \subset A_{k}\right\},
$$




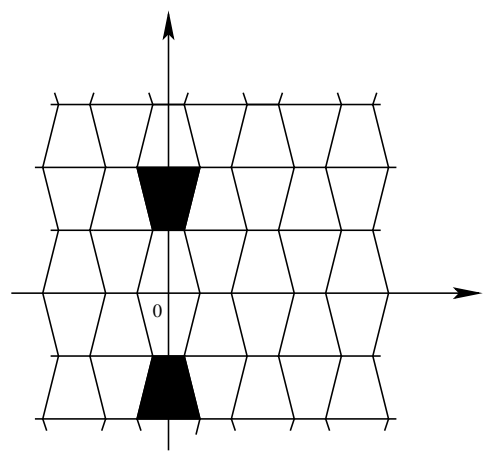

(a)

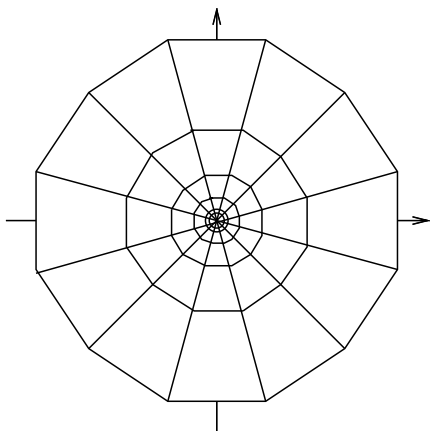

(b)

Figure 3: Examples of tilings (a) $\{A+\{\gamma\}\}_{\gamma \in \Gamma}$ and (b) $\left\{A_{k}\right\}_{k \in \mathcal{K}}$.

and Condition 2 is equivalent to

$$
L^{2}\left(\mathbb{R}^{2}\right)=\bigoplus_{k \in \mathcal{K}} \mathcal{W}_{k}
$$

where the sum is orthogonal. Details of the proof are left to the reader.

Except possibly for one set, the sets $A_{k}$ are obtained by dilating a single set $A$. $\left(A_{k}=D_{k}^{*} A\right.$ where $D_{k}^{*}$ is the adjoint operator of $D_{k}$.) In order to have real valued wavelets, we assume that $A$ is symmetric about 0 , and in order to have a fine angular resolution, $A$ must be included in a cone. An interesting example is given by

$$
A=\left\{\xi \in \mathbb{R}^{2}:\left|\xi_{1}\right|<\alpha\left|\xi_{2}\right| \text { and } 1<\left|\xi_{2}\right|<2\right\},
$$

for some $\alpha>0$. One checks easily that Condition 1 of Proposition 1 is satisfied with $\Gamma^{*}=\mathbb{Z} e_{1}+\mathbb{Z} e_{2}$ where $e_{1}=(0,2)$ and $e_{2}=(3 \alpha, 1)$ (see Fig. 3 -(a)). With good choices for $\alpha$ and $K \in \mathbb{N}$, Condition 2 is satisfied by applying to $A$, dilations of factor 2 (and $1 / 2$ ) and rotations of angle $\pi / K$ (see Fig. 3-(b)). Thus, if $\widehat{\psi^{s}}=2 \pi|A|^{-1 / 2} \chi_{A}$ and $R$ is the rotation of angle $\pi / K$, then

$$
\left\{2^{j} \psi^{s}\left(2^{j} R^{k} \cdot-\gamma\right)\right\}_{j \in \mathbb{Z}, k \in\{1, \cdots, K\}, \gamma \in \Gamma}
$$

is an orthonormal basis for $L^{2}\left(\mathbb{R}^{2}\right)$. There are many other examples that satisfy the conditions of Proposition 1 . The same set $A$ can be dilated in such way that one obtains the tiling of Fig. 2-(c), generating what could be called a Shannon curvelet basis. Notice that, more generally, when going from one scale to a finer one, the number of directions can be multiplied by any integer number. There are also other examples of sets $A$ defining mother wavelets $\psi^{s}$. One has, for instance, $p /(p-1)$-adic directional wavelets, with $p \in \mathbb{N}$, by using

$$
A=\left\{\xi \in \mathbb{R}^{2}:\left|\xi_{1}\right|<\alpha\left|\xi_{2}\right| \text { and } p-1<\left|\xi_{2}\right|<p\right\}
$$

that forms a tiling of $\mathbb{R}^{2}$ when it is shifted along $\Gamma^{*}=\mathbb{Z} e_{1}+\mathbb{Z} e_{2}$ where $e_{1}=(0,2)$ and $e_{2}=((2 p-1) \alpha, 1)$ 


\section{Regular Directional Wavelet Bases}

We address below the construction of regular wavelets (in the sense of wavelets with fast decay) $\left\{\psi_{k}\right\}_{k \in \mathcal{K}}$ that approximate Shannon wavelets $\left\{\psi_{k}^{s}\right\}_{k \in \mathcal{K}}$. The term approximate is used here in the sense that the loss of energy is controlled :

$$
\left\|\psi_{k}-\psi_{k}^{s}\right\|_{2}^{2}<\epsilon, \quad \text { for } \epsilon \text { small. }
$$

Necessary and sufficient conditions for a family $\left\{\psi_{k}(\cdot-\gamma)\right\}_{k \in \mathcal{K}, \gamma \in \Gamma_{k}}$, with general $\psi_{k} \in L^{2}\left(\mathbb{R}^{2}\right)$, to be a basis for $L^{2}\left(\mathbb{R}^{2}\right)$ can be found (see Prop. 4 below). The difficulty is however to find wavelets $\psi_{k}$ that satisfy these conditions. An easy way to construct directional regular wavelets is to consider wavelets implemented in filter bank trees, as their design is reduced to the construction of filter banks. This idea was developed in [13] and several examples of regular directional wavelet basis were given. Close ideas were also proposed in [22]. We develop, in this section, one of the examples introduced in [13]. Numerical results and comparisons are given.

\subsection{Hexagonal Directional Wavelets}

Hexagonal wavelets were introduced in [24] and [9]. These wavelets are obtained one from another by dilation, translation and rotation of $\mp 2 \pi / 3$, and are therefore an example of directional wavelet basis in the sense of [1] with 3 directions. They are designed for images that are sampled on the regular hexagonal lattice

$$
\Gamma=\left(\begin{array}{cc}
1 & 0 \\
1 / \sqrt{3} & 2 / \sqrt{3}
\end{array}\right) \mathbb{Z}^{2} .
$$

Let us recall that the hexagonal reciprocal lattice is

$$
\Gamma^{*}=2 \pi M \mathbb{Z}^{2} \quad \text { where } M=\left(\begin{array}{cc}
1 & -1 / 2 \\
0 & \sqrt{3} / 2
\end{array}\right) .
$$

and that the associated reciprocal cell has the shape of a regular hexagon.

The space $L^{2}\left(\mathbb{R}^{2}\right)$ is decomposed into multiscale and multidirectional subspaces

$$
L^{2}(\mathbb{R})=\bigoplus_{j \in \mathbb{Z}} \bigoplus_{k \in\{1,2,3\}} \mathcal{W}_{j}^{k}=\bigoplus_{j \in \mathbb{Z}} \bigoplus_{k \in\{1,2,3\}} d_{j} R^{k} \mathcal{W},
$$

where $d_{j}$ is the dilation of factor $2^{j}$ and $R$ is the rotation of angle $2 \pi / 3$. Each subspace is spanned by a mother wavelet $\psi_{k}$ that is shifted along an hexagonal lattice at a given scale. More precisely $\left\{2^{j} \psi_{k}\left(2^{j} \cdot-\gamma\right): \gamma \in \Gamma\right\}$ is a Riesz basis or an orthonormal basis for $\mathcal{W}_{j}^{k}$. These wavelets are generated by a multiresolution analysis (MRA) [18] $\left(V_{j}\right)_{j \in J}$ for $L^{2}\left(\mathbb{R}^{2}\right)$ with a scaling function $\phi$ such that $\left\{2^{j} \phi\left(2^{j} \cdot-\lambda\right): \lambda \in \Gamma\right\}$ is a basis for $V_{j}$. The decomposition

$$
V_{j}=V_{j-1} \oplus \mathcal{W}_{j-1}^{1} \oplus \mathcal{W}_{j-1}^{2} \oplus \mathcal{W}_{j-1}^{3}
$$

leads to the equations

$$
\begin{aligned}
\widehat{\phi}(\omega) & =\frac{1}{2} H_{0}(\omega / 2) \widehat{\phi}(\omega / 2), \\
\widehat{\psi_{k}}(\omega) & =\frac{1}{2} H_{k}(\omega / 2) \widehat{\phi}(\omega / 2), \quad \forall k \in\{1,2,3\},
\end{aligned}
$$




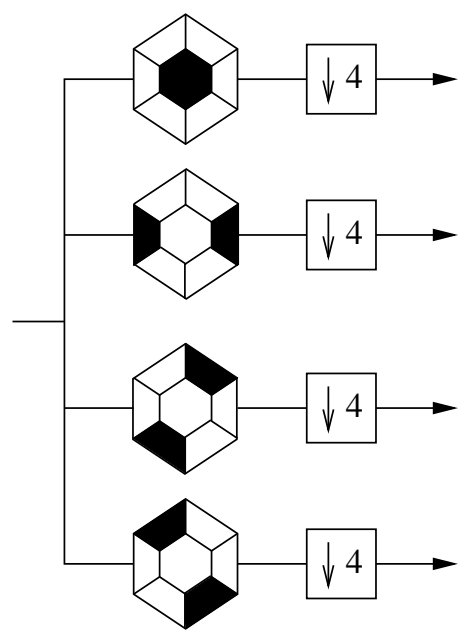

(a)

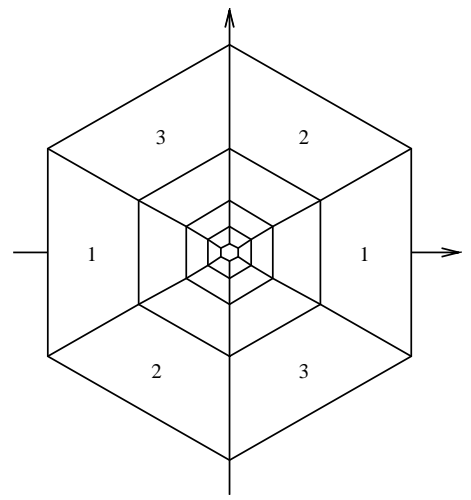

(b)

Figure 4: (a) Hexagonal filter bank and (b) ideal frequency supports of hexagonal wavelets ( $\downarrow 4$ stands for keeping the points of $2 \Gamma$ only).

for almost every $\omega \in \mathbb{R}^{2}$, where $H_{k} \in L^{2}\left(\mathbb{R}^{2} / \Gamma^{*}\right)$, for all $k \in\{0, \ldots, 3\}$. In order to have an orthonormal basis, the conjugate mirror filters $\left(H_{k}\right)_{k \in\{0,1,2,3,\}}$ should be designed in such a way that the matrix

$$
\left(H_{k}(\omega+\gamma)\right)_{k \in\{0,1,2,3\}, \gamma \in\left(0.5 \Gamma^{*}\right) / \Gamma^{*}}
$$

is unitary for almost every $\omega \in \mathbb{R}^{2}$, where

$$
\left(0.5 \Gamma^{*}\right) / \Gamma^{*}=\{(0,0),(\pi, 0),(\pi / 2, \sqrt{3} \pi / 2),(-\pi / 2, \sqrt{3} \pi / 2)\} .
$$

In the case of a discrete image sampled on $\Gamma$, wavelet coefficients are computed via a filter bank tree. The above conjugate mirror filters define a filter bank whose frequency selectivity is symbolized in Figure 4-(a). It decomposes the original image onto 4 subbands. At each level, the same filter bank is applied again to the low frequency subband only. One obtains this way a transform corresponding to the frequency partitioning of Figure 4-(b).

The Hexagonal Directional Wavelet Transform (HDWT) that we propose consists in dividing each directional fluctuation space $\mathcal{W}_{j}^{k}$ in more subspaces with finer directions

$$
\mathcal{W}_{j}^{k}=\bigoplus_{l \in\left\{1, \cdots, 2^{p}\right\}} \mathcal{W}_{j}^{k, l}
$$

This operation is achieved by applying specific filter banks to the high frequency channels of Figure 4-(b). Indeed if we apply the filter bank of Figure 5-(a) to the high frequency subband $\sharp 1$, the latter can be divided into 2 new directional subbands. We can clearly apply the same process to the 2 other high frequency subbands in order to obtain 6 directions as in Figure 6-(a). Notice that the filters used at this step can be designed by dilating and shifting the well known diamond shaped quincunx filter.

Each of these 6 subbands can be divided again into 2 other subbands in order to obtain 12 directions. Apply for example the filter bank of Figure 5-(b) to the subband $\sharp 2$ of Figure 6 -(a). By iterating the process, we can have $3 \times 2^{p}$ directions where $p \in \mathbb{N}$. A parabolic scaling law[5] can be applied to the transform as in Figure 6-(b). 


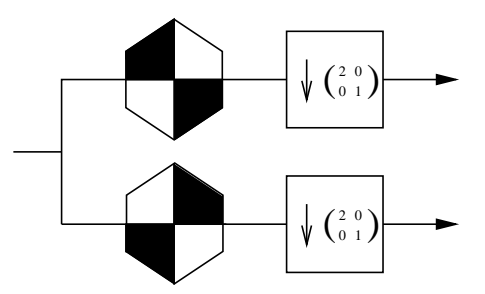

(a)

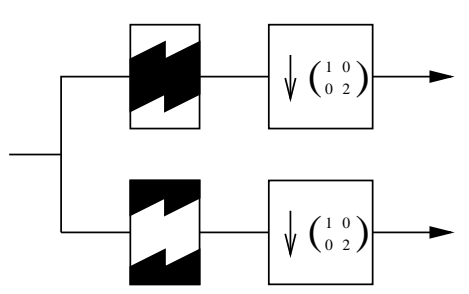

(b)

Figure 5: Two filter banks used in the proposed transform.

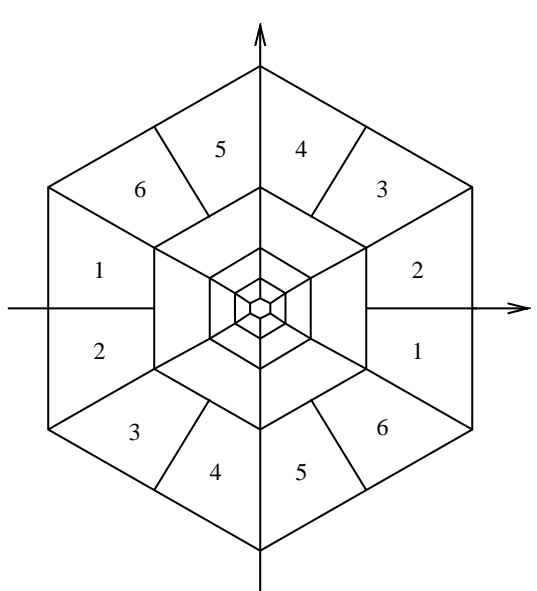

(a)

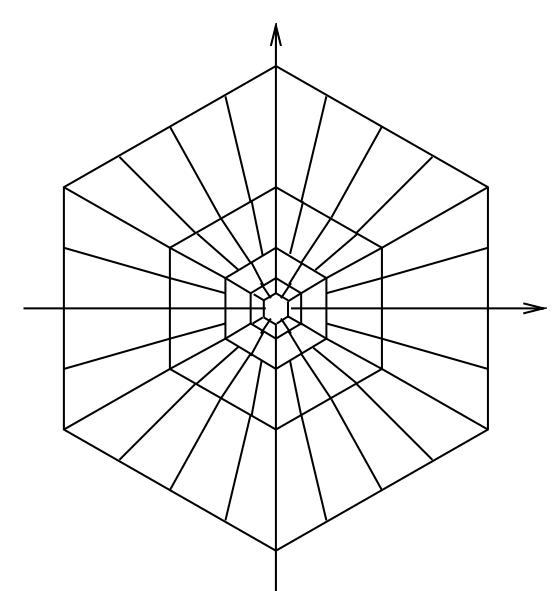

(b)

Figure 6: Ideal frequency supports of hexagonal directional wavelets. 


\subsection{Implementation on square lattice}

The question of filter's design is not developed in this article. Several methods have already been proposed in order to construct non-separable filter banks. Most of them are restricted to 2 channels. For example, generalized McClellan transform [26] permits to design transfer functions whose essential support approximate some arbitrary set. In [9], a 4-channel biorthogonal, hexagonal filter bank was constructed. More recently, a method has been proposed to obtain 2-channel filter banks with directional vanishing moments [10]. However the design of M-band filters with finite impulse responses and an arbitrarily small angle between two consecutive directional vanishing moments remains an open question.

As our objective is to construct wavelets that approximate the associated Shannon wavelets, we do not use the hexagonal filter of [9] that is not sufficiently well localized in the frequency domain. In the experiments below, all the filters involved in the transform have been designed using the equation

$$
H_{0}=\frac{|\operatorname{det} D|^{1 / 2} g * \chi_{A_{0}}}{\left(\sum_{\gamma \in\left(D^{-1} \Lambda^{*}\right) / \Lambda^{*}}\left|g * \chi_{A_{0}}(.+\gamma)\right|^{2}\right)^{1 / 2}},
$$

where $A_{0}$ is the ideal support of $H_{0}, D$ is the dilation matrix of the filter bank and $\Lambda$ is the lattice that supports the signal to be filtered. We chose for $g$ a Gaussian function. It is approximated by a compactly supported function. Such a design method is a simple way to obtain simultaneously orthonormality, space and frequency localization, linear phase and nearly vanishing directional moments. Moreover, when $g$ tends to a Dirac measure $\delta_{0}, H_{0}$ tend to the associated Shannon filters in the sense that

$$
\left\|H_{0}-|\operatorname{det} D|^{1 / 2} \chi_{A_{0}}\right\|_{2} \rightarrow 0 \text {. }
$$

In other words, Condition (1) can be satisfied for arbitrarily small $\epsilon$.

These filters have however infinite impulse responses. They are therefore implemented in the Fourier domain. It may seems to be a drawback of the proposed implementations. There are however several reasons for using the Fourier transform. First, in order to have a fine directionality one should use filters that are very well localized in the frequency domain and that have therefore large space support. If the associated filter bank is implemented in the spatial domain, the computation time of the algorithm is proportional to the length of that support and might be larger than the computation time of a FFT (although it is $O(N)$ where $N$ is the size of the image). Secondly, if $O(\log N)$ directions are set at the finest scale, the computation time of the whole transform that is implemented in the spatial domain is actually $O(N \log N)$. A major argument for using the Fourier transform is that images are in general sampled on a square lattice. The Fourier transform is a natural tool to re-sample images on an hexagonal lattice as it comes down to shifting their spectrum. This point is developed further below. At last recall that the curvelets are also bandlimited and are implemented in the Fourier domain. In some sense, the transform remains faithful to the curvelets although it generates some aliasing problems that are addressed later. By using bandlimited wavelets, we can measure in some way the magnitude of this aliasing.

As it is alluded to above, the algorithm is implemented for images that are sampled on the square lattice $\mathbb{Z}^{2}$. There are at least two ways to implement hexagonal directional wavelets on a square lattice. A first method consists in changing the filters $H_{k}$ into $H_{k} \circ M$ where $M$ is given in (2). If one assumes that the spectrum of 


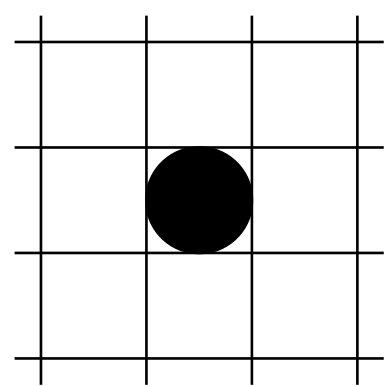

(a)

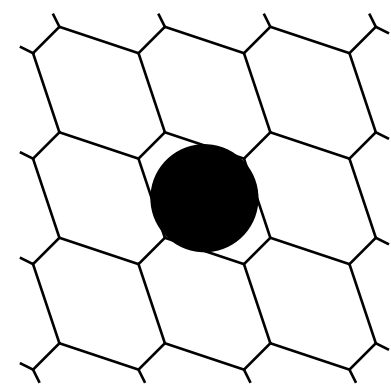

(b)

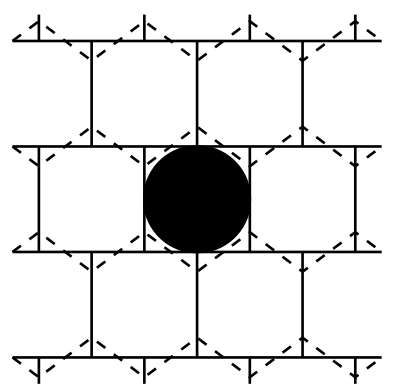

(c)

Figure 7: Two possible reciprocal cells for the square lattice, (a): Voronoi cell and (b): irregular hexagonal cell. Periodization of a square reciprocal cell, (a): square lattice. (c) : irregular hexagonal lattice. The dashed lines corresponds to the Voronoi set of the hexagonal reciprocal lattice. The black disk is the essential frequency support of an image.

images is supported on the circumscribed disk of the square reciprocal cell, then there is nearly no aliasing in the obtained transform as it is shown in Figure 7-(b). The transform can moreover be implemented with finite impulse response filters. However the obtained refinement filter is clearly not enough isotropic.

In the experiments below, we follow another approach. A standard Fourier transform is applied to the image. The obtained transform is however considered as the Fourier transform of an image sampled on the irregular hexagonal lattice

$$
\widetilde{\Gamma}=\left(\begin{array}{cc}
1 & 0 \\
1 / 2 & 1
\end{array}\right) \mathbb{Z}^{2}
$$

It amounts to exchanging the periodization vectors $(2 \pi, 0)$ and $(0,2 \pi)$ for $(2 \pi, 0)$ and $(\pi, 2 \pi)$. The Fourier coefficients remain the same, they are just shifted. As it is shown in Figure 7-(c), one can keep the same reciprocal cell.

Notice that the image could also be re-sampled on a regular hexagonal lattice without deteriorating the circular spectrum of the image. The number of samples to represent the image would be reduced that way by a factor $\sqrt{3} / 2$. This idea is not developed in the article as our objective is to design bases for $\ell^{2}\left(\mathbb{Z}^{2}\right)$. The reciprocal cells of Figure 7 have the same surface. Thus there is no lost of data when exchanging the lattices whatever the shape of the frequency support of the image. Moreover this process transforms an orthonormal basis into an orthonormal basis.

All the filter banks are implemented in the Fourier domain. Let $X$ be the Fourier transform of an input, discrete 2D signal $x$. For each filter bank, the output $y_{k}$ of the $k^{\text {th }}$ channel is given by its Fourier transform

$$
Y_{k}=\sum_{\gamma \in\left(D^{-1} \Lambda^{*}\right) / \Lambda^{*}} H_{k}(\cdot+\gamma) X(\cdot+\gamma) .
$$

At the end of the process, an inverse Fourier transform is applied to each subband. A different Fourier transform is used for each lattice. They all run in an $O(N \log N)$ algorithm for an image of size $N$ (see [19] for an hexagonal FFT, and the appendix for a FFT on a general, regular lattice).

The hexagonal filter is designed using (3) with

$$
A_{0}=\left\{\omega \in \mathbb{R}^{2}:\left|\omega_{1}\right|<\pi / 2 \&\left|2 \omega_{1}+3 \omega_{2}\right|<2 \pi \&\left|2 \omega_{1}-3 \omega_{2}\right|<2 \pi\right\}+\tilde{\Gamma}^{*},
$$




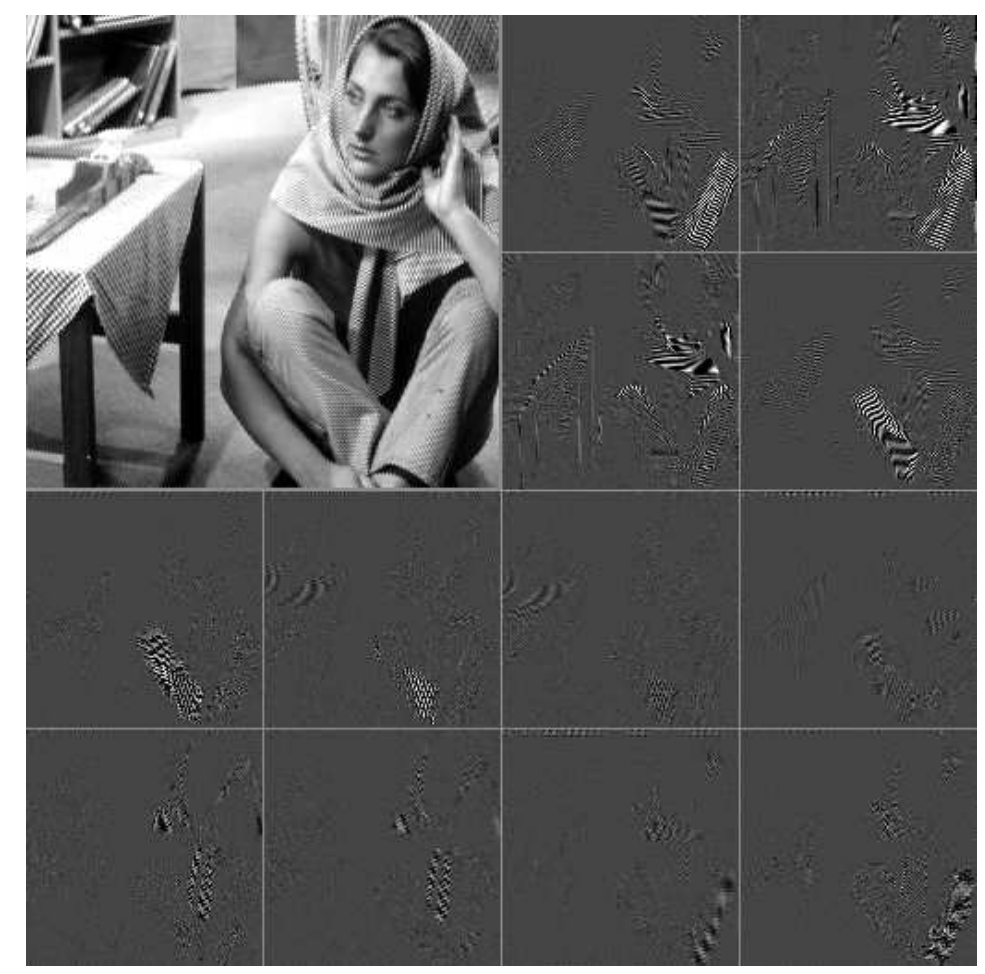

Figure 8: Decomposition of the Barbara image onto 12 directional subbands.

$\Lambda=\widetilde{\Gamma}, D=2 I d_{2}$ and $\left(0.5 \tilde{\Gamma}^{*}\right) / \tilde{\Gamma}^{*}=\{(0,0),(\pi, 0),(\pi / 2, \pi),(-\pi / 2, \pi)\}$. The wavelet filters are defined by

$$
\begin{aligned}
& H_{1}(\omega)=e^{i\left(\omega_{1}-\omega_{2} / 2\right)} H_{0}(\omega+(\pi, 0)), \\
& H_{2}(\omega)=e^{i\left(\omega_{1}+\omega_{2} / 2\right)} H_{0}(\omega+(\pi / 2, \pi)), \\
& H_{3}(\omega)=e^{i \omega_{2}} H_{0}(\omega+(-\pi / 2, \pi)) .
\end{aligned}
$$

One checks easily that the associated matrix is unitary. The 2-channel filter banks are designed by the same method. One set

$$
H_{1}(\omega)=e^{i\langle\omega, \delta\rangle} H_{0}(\omega+\gamma)
$$

where $\{\gamma\}=\left(D^{-1} \Lambda^{*}\right) / \Lambda^{*}$ and $\delta$ is such that $\langle\gamma, \delta\rangle=\pi$ modulo $2 \pi$.

The hexagonal directional filter banks have been implemented up to 12 directions. Figure 8 shows the Barbara image that have decomposed onto 12 directional subbands and a low frequency subband.

\subsection{Numerical results}

We present in this section some numerical results obtained with the HDWT, and comparisons with the Hexagonal Wavelet Transform (HWT) and the $9 / 7$ Separable Wavelet Transform (SWT) [8]. Let us point out that the compression performance of the $9 / 7$ tap filters are widely recognized and that it has been incorporated in the JPEG 2000 standard. On the other hand, we do not show the best possible implementation 


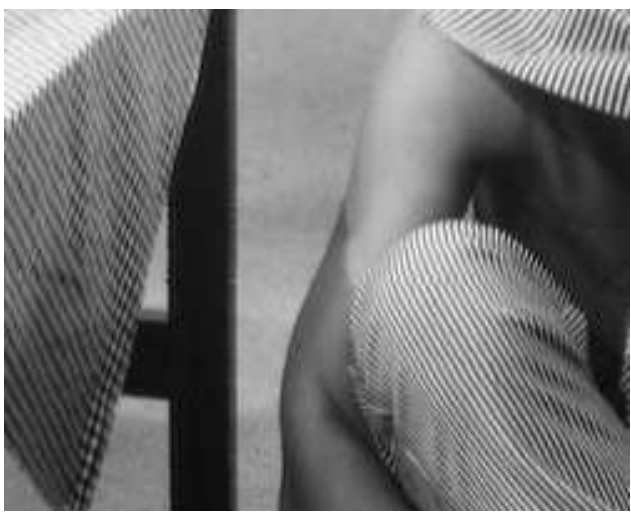

(a)

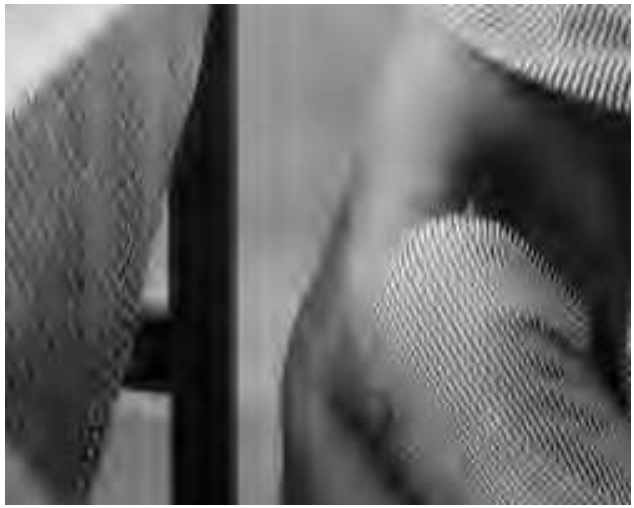

(c)

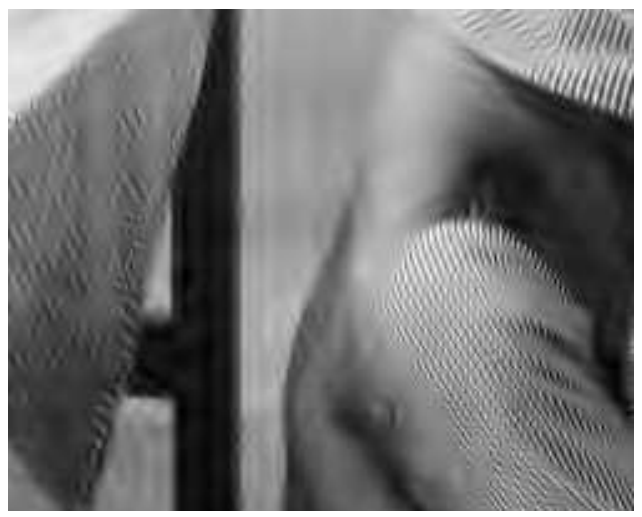

(b)

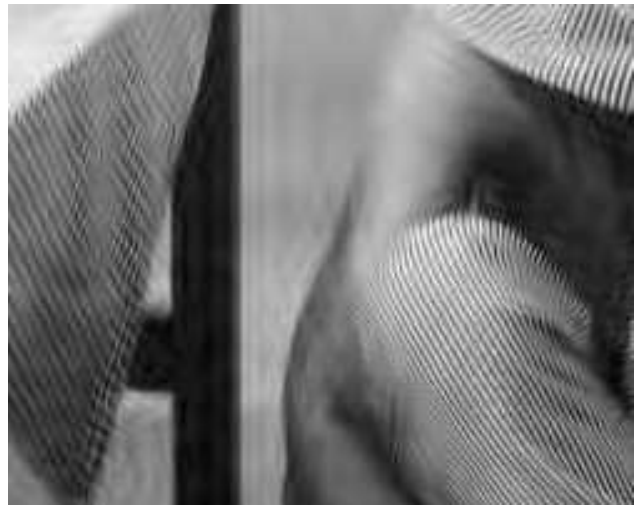

(d)

Figure 9: Non linear approximation of Barbara image (detail). (a) Original image. (b) HWT (PSNR = 27.08). (c) SWT (PSNR = 27.02). (d) HDWT (PSNR = 28.58). Images are reconstructed from the $10^{4}$ largest coefficients.

of the transform proposed in Section 3.1. For instance, using finite impulse response filters on hexagonally sampled images would permit to reduce the Gibbs oscillations generated by the thresholding or quantization of the coefficients. The 3 transforms are implemented with 6 levels of decomposition. There are 12 directions at the 2 finest scales of the HDWT, 6 at the 2 intermediate scales and 3 at the coarsest scales. The HWT consists in choosing 3 directions at each level of the HDWT.

In Figure 9 we show non linear approximations of the $512 \times 512$ Barbara image. For the 3 transforms, the $10^{4}$ largest coefficients have been retained. We obtained a better result with the HDWT than with the SWT and HWT, in terms of PSNR and visually. As expected, textures are better preserved. Statistical comparisons are given in Figure 10. When few coefficient are retained the HDWT gives a much better approximation than the SWT. The two lines merge when more than $10^{5}$ coefficients are retained. However the SWT never outperforms the HDWT contrary to what happens with redundant frames such as curvelets and contourlets.

As alluded to in the introduction, the benefit of directional transforms is larger in regions where the image has a strong and smooth geometrical flow. The Barbara image is therefore very well adapted to the HDWT. When the comparison is made on 


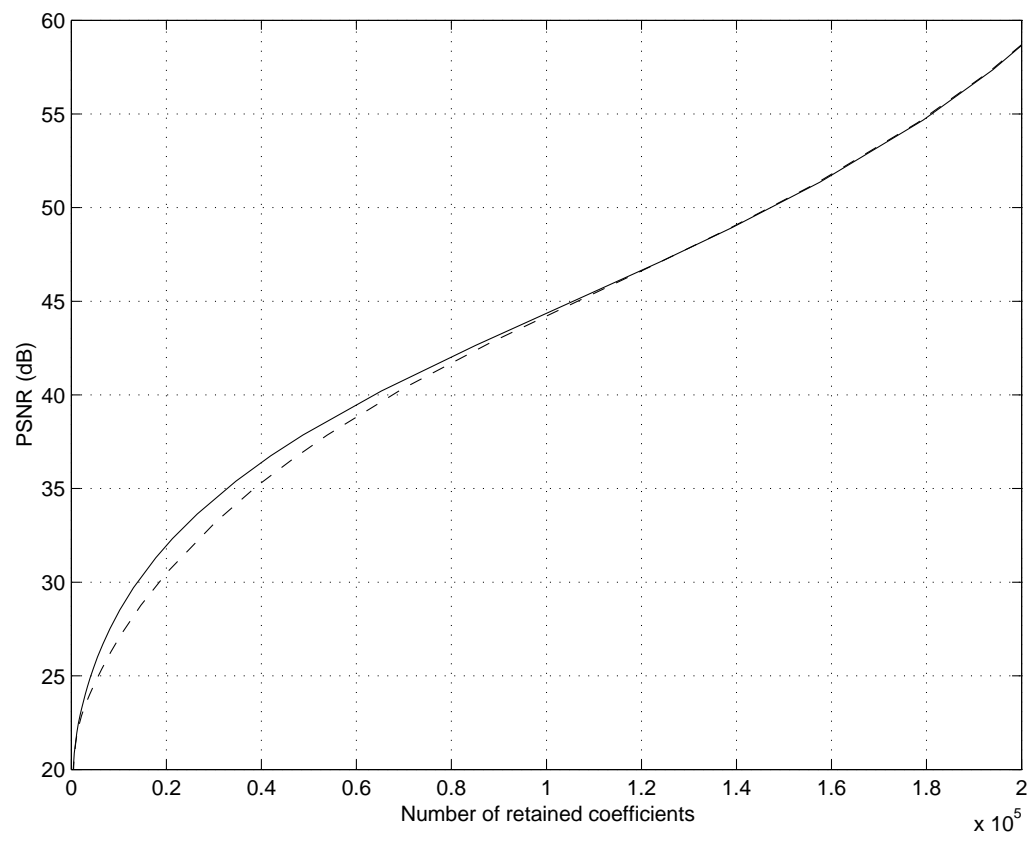

Figure 10: PSNR versus number of retained coefficients for Barbara image (dashed line: SWT, solid line: HDWT).

texture-less images, the improvement becomes less significant. For instance, when the 3 transforms are applied to the $512 \times 512$ fresco image and the $10^{4}$ largest coefficients are retained, the PSNR of the obtained images are close (see Figure 11). We emphasize that the efficiency of the HDWT could be improved by designing new filter banks. The proposed transform has however a drawback that appears clearly when we look at the detail shown in Figure 11. Although the HWT does not have ambiguous directions, it has only 3 directions. As a consequence, some directions are not well reproduced. With the configuration we adopted, this is the case for the horizontal structures that appears on the right of the image. As expected, only the horizontal and vertical structures are well preserved with the SWT. All the directions should be well represented with the HDWT. The left hand side of the image is better rendered with the HDWT than with the HWT and the SWT. But one can observe that the horizontal structures on the right hand side are not well preserved with the HDWT. This could be justified by the fact that, like the HWT, the HDWT has only 3 directions at the coarsest scales. However the 12 directions at the finest scales should make up for the lack of directions of coarse wavelets. The main reason for this phenomenon is that some directional wavelets are aliased. This problem is addressed in the next section. 


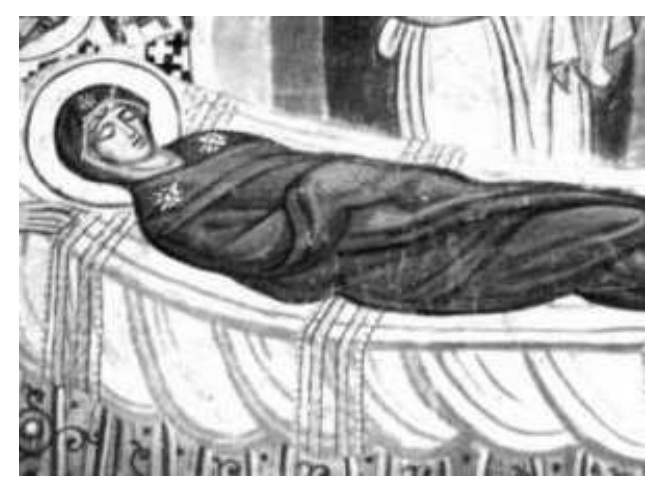

(a)

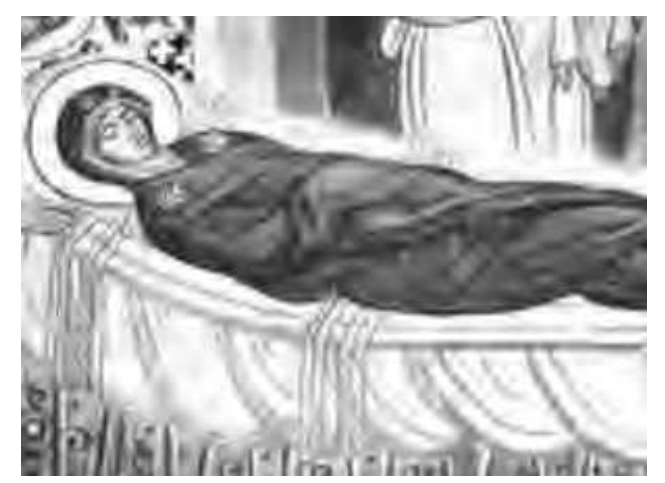

(c)

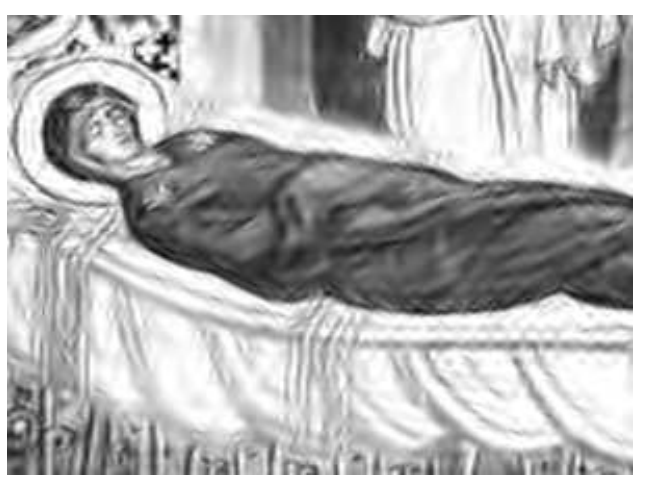

(b)

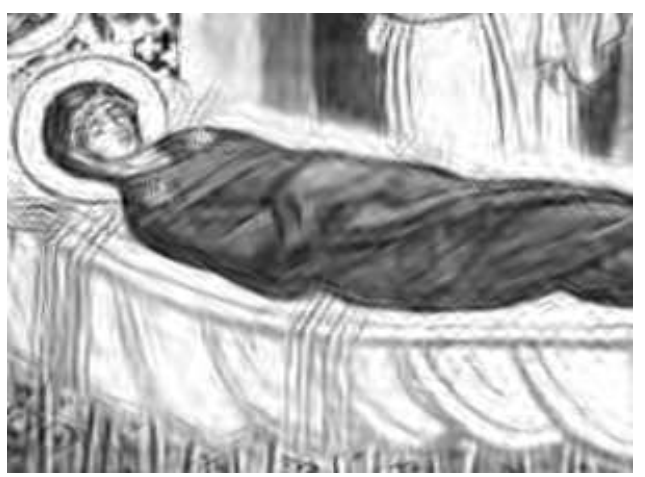

(d)

Figure 11: Non linear approximation of fresco image (detail). (a) Original image. (b) HWT $(\mathrm{PSNR}=24.85)$. (c) SWT $(\mathrm{PSNR}=24.87)$. (d) HDWT $(\mathrm{PSNR}=25.03)$. Images are reconstructed from the $10^{4}$ largest coefficients. The 3 images are aliased but in different directions

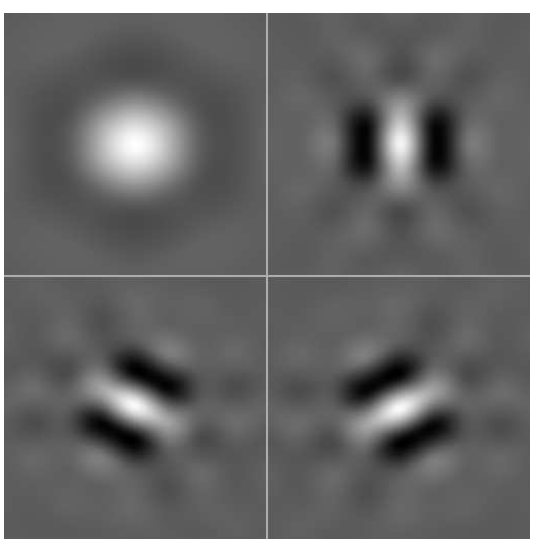

(a)

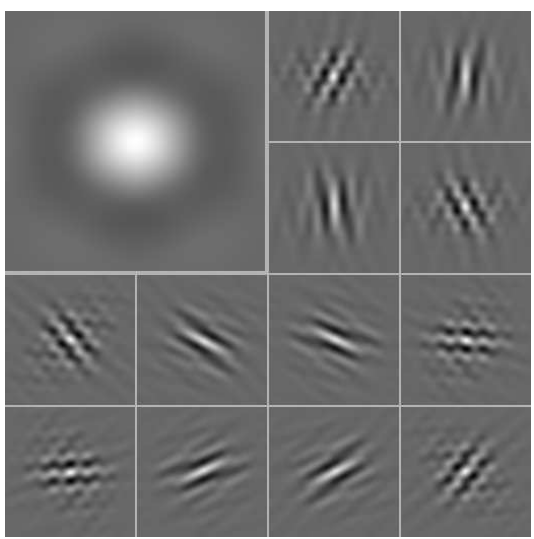

(b)

Figure 12: (a) Hexagonal wavelets and (b) hexagonal directional wavelets. The hexagonal directional wavelets are aliased. 


\section{On the frequency localization of Directional Wavelet Bases}

\subsection{Frequency localization of the HDWT}

The aliasing alluded to above appears clearly on 6 of the wavelets shown in Figure $12^{1}$, in the case of 12 directions. More precisely, the 12 wavelets are aliased. Each of them has 2 directions, the main direction that corresponds to the ideal frequency support, and an aliased direction obtained by symmetry about the Cartesian axes. This phenomenon could be justified by the way the HDWT is implemented, that is to say by the combinations, at each scale, of an hexagonal filter bank and one or more 2-channel filter banks. This combination generates non-uniform filter banks [13] that split the image into a refinement subband and, for example, 12 directional wavelet subbands as in Figure 8, and whose wavelet filters are aliased. Figure 13 shows the transfer functions of 2 among the 12 passband directional wavelet filters that are designed by the proposed method. Some spikes appear clearly in the right hand side graph. They do not appears in the left hand side one because the main direction and the aliased direction are too close.

The causes for this frequency leaks are unfortunately not due to the way the 12 subbands are split. Suppose indeed that we want to compute directly the wavelet coefficients at a same scale, using a single non-uniform filter bank, and that we design therefore new filters. Then the obtained filters will be aliased again, for two reasons. First, the periodization of the transfer functions introduces energy in unwanted regions of the spectrum. It corresponds to the 2 largest peaks in the aliased direction, in Figure 13-(b). The same phenomenon appears in some other transforms based on filter banks such as decimated contourlets.

Another reason why the hexagonal directional wavelets are aliased is connected to the permissibility condition for the filter banks that are used in this transform. One says that a filter bank is (or more precisely the passband supports of its filters are) permissible if one can design smooth transfer functions whose supports remain in a neighborhood of their ideal supports [7], or in other words, if for all $\epsilon>0$, there exists a perfect reconstruction filter bank $\left\{H_{k}\right\}_{k}$ such that supp $H_{k} \subset A_{k}+B(0, \epsilon), \forall k$, where $B(0, \epsilon)$ stands for the ball of center 0 and radius $\epsilon$, and $A_{k}$ is the ideal frequency support of the filter. Although each uniform (2-channel and 4-channel) filter bank used in the proposed transform is permissible, the non uniform filter banks generated by the combination of these filter banks are not permissible. The frequency support of the filter of Figure 13-(b) is schematized in Figure 14-(b). In Figure 14-(a) is shown the frequency support that would have a permissible filter if such a filter existed. Notice that by the periodization of the transfer function, the ideal filter is also aliased. A detailed proof of this property is given in [13] for another frequency partitioning. It can be easily adapted to the HDWT. Moreover, the arguments developed in the proof of Proposition 2 below can be applied to show the non-permissibility of directional filter banks.

\footnotetext{
${ }^{1}$ We do not show the discrete wavelets at the finest scale of the HDWT, but the wavelets defined on a continuous domain
} 

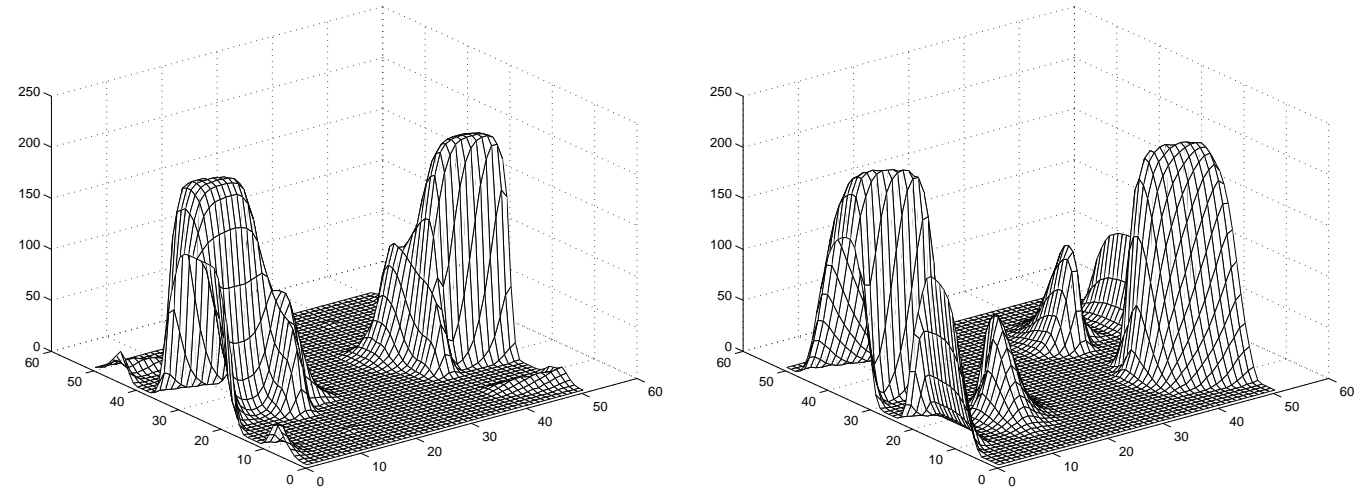

Figure 13: Transfer functions of 2 directional wavelet filters.

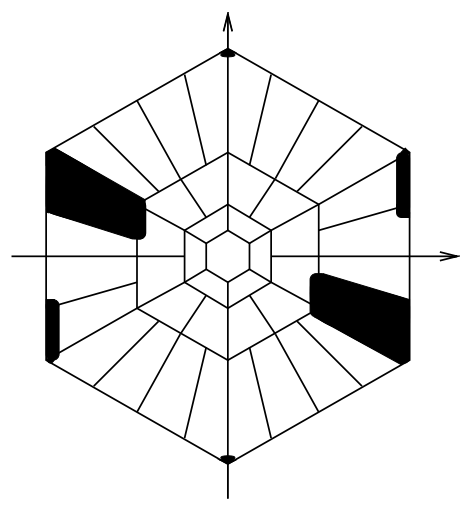

(a)

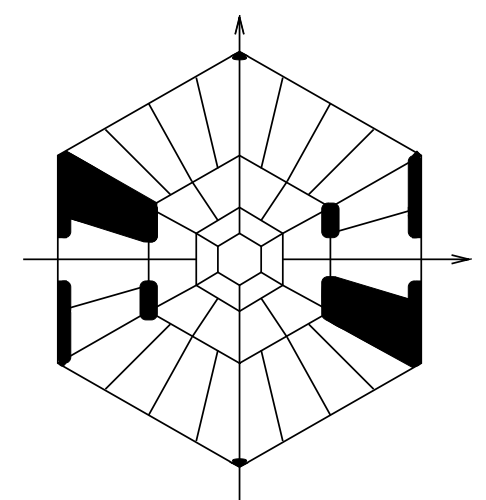

(b)

Figure 14: (a) Ideal and (b) real frequency supports of passband hexagonal directional filters. 


\subsection{Frequency localization of general directional wavelets}

From this argumentation, one might think that the obstruction to the construction of non-aliased directional wavelet bases could be bypassed if one uses wavelets that are not generated by a filter bank tree. (Recall that the periodization of transfer functions introduces aliasing.) Consider a Shannon directional wavelet basis $\left\{\psi_{k}^{s}(\cdot-\right.$ $\gamma)\}_{k \in \mathcal{K}, \gamma \in \Gamma_{k}}$ that we want to approximate with regular wavelets. Let us suppose that the basis satisfies a parabolic scaling law so that the number of directions increases with the scale. Recall that this last property is fundamental to obtain an optimal sparsity behavior for images with $C^{2}$ singularities [6]. The frequency support of each wavelet is adjacent to the support of 2 other wavelets that have the same scale and at least 1 wavelet that has a coarser scale. If the number of directions increases with the scale, there is also a wavelet whose frequency support is adjacent to the support of at least 2 wavelets that have a finer scale. There are therefore at least 5 neighboring wavelets. We claim that this Shannon wavelet basis cannot be approximated by a regular wavelet basis $\left\{\psi_{k}(\cdot-\gamma)\right\}_{k \in \mathcal{K}, \gamma \in \Gamma_{k}}$ so that the frequency support of $\psi_{k}$ remains in an arbitrary small neighborhood of $\operatorname{supp} \widehat{\psi_{k}^{s}}$, for all $k \in \mathcal{K}$. We obtain this way a generalization of the property on the non-permissibility of the filter banks, discussed above.

Proposition 2 Consider a Shannon directional wavelet basis $\left\{\psi_{k}^{s}(\cdot-\gamma)\right\}_{k \in \mathcal{K}, \gamma \in \Gamma_{k}}$ with $\widehat{\psi_{k}^{s}}=\left|A_{k}\right|^{-1 / 2} \chi_{A_{k}}$, such that one of the sets $A_{k}$ has more than 4 adjacent sets $A_{l}$. For $\epsilon>0$ sufficiently small, there are no families of functions $\left\{\psi_{k}\right\}_{k \in \mathcal{K}}$ and $\left\{\widetilde{\psi}_{k}\right\}_{k \in \mathcal{K}}$ that satisfy the following properties simultaneously.

i) $\left\{\psi_{k}(\cdot-\gamma)\right\}_{k \in \mathcal{K}, \gamma \in \Gamma_{k}}$ and $\left\{\widetilde{\psi}_{k}(\cdot-\gamma)\right\}_{k \in \mathcal{K}, \gamma \in \Gamma_{k}}$ are biorthogonal bases for $L^{2}\left(\mathbb{R}^{2}\right)$.

ii) $\widehat{\psi_{k}}$ and $\widehat{\bar{\psi}_{k}}$ are continuous, $\forall k \in \mathcal{K}$.

iii) supp $\widehat{\psi_{k}} \subset A_{k}+B\left(0, \epsilon \operatorname{diam}\left(A_{k}\right)\right)$ and supp $\widehat{\widetilde{\psi}_{k}} \subset A_{k}+B\left(0, \epsilon \operatorname{diam}\left(A_{k}\right)\right), \forall k \in \mathcal{K}$.

We tried to give a statement that applies to the most general frequency partitionings. In the proof, we consider 6 wavelets only, and in condition (iii), the radius of the ball does not have to depend on the diameter of $A_{k}$. In the case when $A_{k}=D_{k}^{-1} A$, it is however natural to assume that supp $\widehat{\psi_{k}} \subset D_{K}^{-1}(A+B(0, \epsilon))$.

Proof. The proof by contradiction of this statement is based on geometric arguments. Suppose Conditions i), ii) and iii) are satisfied for an $\epsilon>0$ arbitrarily small. Denote by $A_{0}$ the frequency support of a Shannon wavelet having at least 5 neighboring wavelets, and by $A_{k}, k \in\{1, \cdots, 5\}$, the 5 neighboring wavelet frequency supports (see Fig. 15). The orthogonality condition

$$
\left\langle\psi_{0}(\cdot-\gamma), \widetilde{\psi}_{k}\right\rangle=0, \quad \forall \gamma \in \Gamma_{0}, \forall k \in\{1, \cdots, 5\}
$$

becomes, after a standard calculation,

$$
\sum_{\gamma \in \Gamma_{0}^{*}} \widehat{\widehat{\psi_{0}}(\cdot-\gamma)} \widehat{\widetilde{\psi}_{k}}(\cdot-\gamma)=0, \quad \forall k \in\{1, \cdots, 5\},
$$

while the condition

$$
\left\langle\psi_{0}(\cdot-\gamma), \widetilde{\psi}_{0}\right\rangle=\left\{\begin{array}{lll}
1 & \text { if } \quad \gamma=0 \\
0 & \text { if } \quad \gamma \in \Gamma_{0} \backslash\{0\}
\end{array}\right.
$$




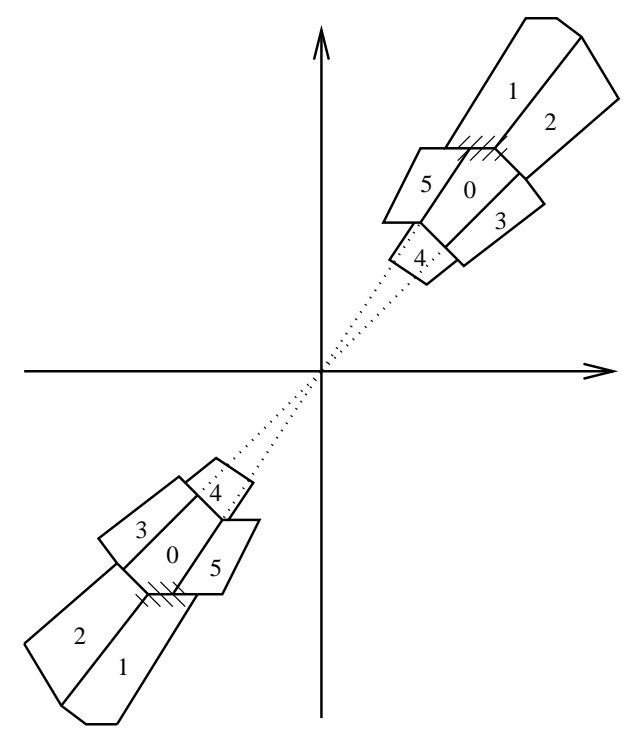

Figure 15: Ideal frequency support of $\psi_{k}$ for $k \in\{0, \cdots, 5\}$. The hatched areas correspond to the support of $\overline{\bar{\psi}_{0}} \widehat{\widetilde{\psi}_{1}}$.

leads to

$$
\sum_{\gamma \in \Gamma_{0}^{*}} \overline{\widehat{\psi}_{0}}(\cdot-\gamma) \widehat{\psi_{0}}(\cdot-\gamma)=1
$$

where $\bar{z}$ stands for the conjugate complex number of $z$. If Conditions ii) and iii) are satisfied for $\epsilon$ small enough, then the support of $\overline{\bar{\psi}_{0}} \widehat{\widetilde{\psi}_{k}}$ has 2 connected components. They are represented by the hatched areas in Figure 15, when $k=1$. By (4), this 2 components should cancel each other out when $\widehat{\widehat{\psi}_{0}} \widehat{\widetilde{\psi}_{k}}$ is shifted along $\Gamma_{0}^{*}$. The reciprocal lattice $\Gamma_{0}^{*}$ must also satisfy (5) which means that the ideal support of $\widehat{\psi_{0}}$ forms a tiling of $\mathbb{R}^{2}$ when it is shifted along $\Gamma_{0}^{*}$. These last 2 conditions are summed up in Figure 16-(a). We can observe that, in this example, the 2 components cancels each other out when $k=1$ but does not when $k=2$ (see Figure 16-(b)).

More generally, there does not exists a tiling $\left\{A_{0}+\{\gamma\}\right\}_{\gamma \in \Gamma_{0}^{*}}$ for the ideal support of $\widehat{\psi_{0}}$ such that the 5 (or more) neighboring wavelets $\widehat{\psi_{k}}$ satisfy Condition (4) in the sense that the 2 connected components of $\widehat{\widehat{\psi_{0}}} \widehat{\bar{\psi}_{k}}$ can cancel each other out. Indeed, the tiling generated by the support of $\widehat{\psi_{0}}$ is an isohedral tiling [15]. (It is generated by a single tile and its symmetry group acts transitively on the tiles.) Among the 81 types of possible isohedral tilings, several ones have tiles with more than 4 adjacent tiles but one can verify that none of them has the required type. An easy way to check this claim is to consider a tile formed by 2 adjacent tiles, as for instance, the tile marked by 0 in Figure 16. When it is shifted along the good regular lattice, it generates another isohedral tiling. If it has the required type, the tile should have at least 8 adjacent tiles as in Figure 17. The conclusion comes from the fact that a tile of an isohedral tiling has at most 6 adjacent tiles [15]. 

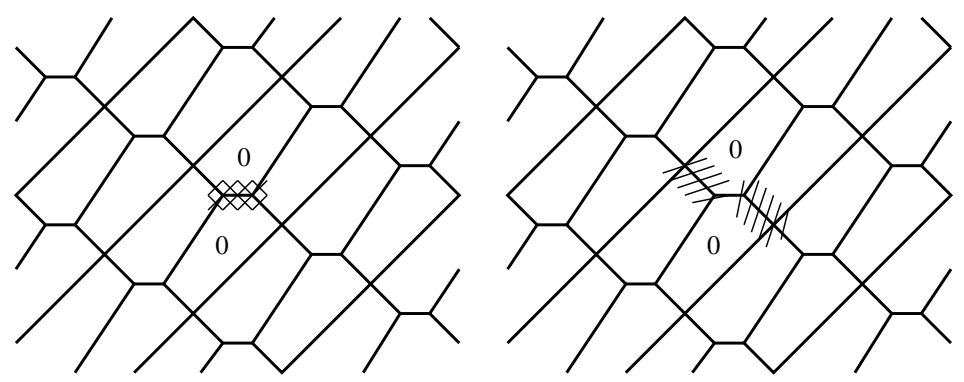

Figure 16: Ideal support of $\widehat{\psi_{0}}(\cdot-\gamma)$ for $\gamma \in \Gamma_{0}^{*}$. The 2 components of the support of $\widehat{\bar{\psi}_{0}(\cdot-\gamma)} \widehat{\widetilde{\psi}}_{1}(\cdot-\gamma)$ cancel each other out while the components of the support of $\widehat{\widehat{\psi}_{0}}(\cdot-\gamma) \widetilde{\psi_{2}}(\cdot-\gamma)$ do not.
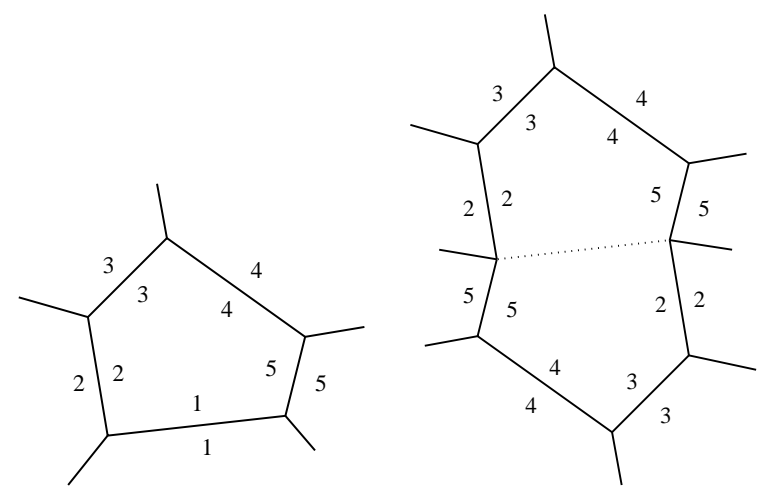

Figure 17: The required type of tiling does not exist since tiles of an isohedral tiling have at most 6 adjacent tiles. 
Proposition 2 does not apply to the frequency partitioning of Figure 3-b as each set $A_{k}$ has only 4 adjacent sets. The argumentation above can however be generalized to this example if one includes sets that are connected by a dot, though the aliasing might remain localized in the neighborhood of a dot. This phenomenon occurs with hexagonal wavelets but that the artifact is visually negligible (see Figure 12-(a)). Notice that there are also non-aliased bases that satisfy a non isotropic scaling law but that are not obtained by shifting a finite number of mother wavelets at each scale. One might think here of orthonormal ridgelets [12].

\section{Directional Wavelet Tight Frames With Arbitrary Small Redundancy Factor}

Proposition 2 reveals an obstruction to the construction of directional wavelet bases that approximate properly Shannon directional wavelets. On the other hand, second generation discrete curvelets [3] approximate the associated Shannon wavelet tight frame with a good frequency localization. This frame is however highly redundant. We show in this section that the latter construction can be generalized in order to obtain well localized directional wavelet tight frames with arbitrary small redundancy factor.

\subsection{Shannon Directional Wavelet Tight Frames}

Proposition 1 can be generalized to the case of tight frames as follows.

Proposition 3 Let $\widehat{\psi_{k}^{s}}=\left(1 /\left|A_{k}\right|\right)^{1 / 2} \chi_{A_{k}}$ with $A_{k} \subset \mathbb{R}^{2}$, for all $k \in \mathcal{K}$. If

1. $\left\{A_{k}+\{\gamma\}\right\}_{\gamma \in \Gamma_{k}^{*}}$ is a packing of $\mathbb{R}^{2}$,

2. $\left\{A_{k}\right\}_{k \in \mathcal{K}}$ is a tiling of $\mathbb{R}^{2}$,

3. $\frac{\left|\mathcal{C}_{k}\right|}{\left|A_{k}\right|}=\rho(\geq 1), \forall k \in \mathcal{K}$,

where $\left|\mathcal{C}_{k}\right|$ is the area of the reciprocal cell of $\Gamma_{k}$, then the family $\left\{\psi_{k}^{s}(\cdot-\gamma)\right\}_{k \in \mathcal{K}, \gamma \in \Gamma_{k}}$ is a tight frame for $L^{2}\left(\mathbb{R}^{2}\right)$ with redundancy factor $\rho$.

Proof. Condition 1 of Proposition 1 has been changed in Conditions 1 and 3. They are sufficient conditions for $\left\{\psi_{k}^{s}(\cdot-\gamma)\right\}_{k \in \mathcal{K}, \gamma \in \Gamma_{k}}$ to be a tight frame for $\mathcal{W}_{k}$ with redundancy factor $\rho$.

\subsection{Regular Directional Wavelet Tight Frames}

In the discussion below, we assume that the functions $\widehat{\psi_{k}}$ have fast decay and are bandlimited. We make therefore the assumption that $\psi_{k}$ is in the Schwartz class $\mathcal{S}\left(\mathbb{R}^{2}\right)$ although Proposition 4 could be proved with more general functions. This hypothesis makes however convergence questions easier to treat. 
Proposition 4 Suppose that $\psi_{k} \in \mathcal{S}\left(\mathbb{R}^{2}\right),\left\|\psi_{k}\right\|_{2}=1, \forall k \in \mathcal{K}$,

$$
\left\|\sum_{k \in \mathcal{K}}\left|\mathcal{C}_{k}\right|\left|\widehat{\psi_{k}}\right|^{2}\right\|_{\infty}<\infty
$$

and

$$
\min \left\{|x-y|: x, y \in \bigcup_{k \in \mathcal{K}} \Gamma_{k}^{*} \& x \neq y\right\}>0 .
$$

The family $\left\{\psi_{k}(\cdot-\gamma)\right\}_{k \in \mathcal{K}, \gamma \in \Gamma_{k}}$ is a tight frame for $L^{2}\left(\mathbb{R}^{2}\right)$ with redundancy factor $\rho$ if and only if

a. $\sum_{k \in \mathcal{K}}\left|\mathcal{C}_{k}\right|\left|\widehat{\psi_{k}}(\xi)\right|^{2}=(2 \pi)^{2} \rho$,

b. $\sum_{k \in \mathcal{K}_{\gamma}}\left|\mathcal{C}_{k}\right| \widehat{\widehat{\psi_{k}}}(\xi-\gamma) \widehat{\psi_{k}}(\xi)=0, \quad \forall \gamma \in \bigcup_{k \in \mathcal{K}} \Gamma_{k}^{*}$,

where $\mathcal{K}_{\gamma}=\left\{k \in \mathcal{K}: \gamma \in \Gamma_{k}^{*}\right\}$.

Notice that the condition $\min \left\{|x-y|: x, y \in \bigcup_{k \in \mathcal{K}} \Gamma_{k}^{*} \& x \neq y\right\}>0$ is not satisfied by directional wavelets that are generated by standard rotations and dilations as considered in [1] and shown in Figure 3-(b). In this case, another proposition needs to be proved. On the other hand, this condition is satisfied by all the directional wavelets that fulfill a parabolic scaling law and such that low frequencies are represented by a scaling function $\phi$. It is therefore satisfied by curvelets.

Proof. Let us assume that $\left\{\psi_{k}(\cdot-\gamma)\right\}_{k \in \mathcal{K}, \gamma \in \Gamma_{k}}$ is a tight frame for $L^{2}\left(\mathbb{R}^{2}\right)$ with redundancy factor $\rho$. It is characterized by the equation

$$
\rho f(x)=\sum_{k \in \mathcal{K}} \sum_{\gamma \in \Gamma_{k}}\left\langle f, \psi_{k}(\cdot-\gamma)\right\rangle \psi_{k}(x-\gamma),
$$

for all $f \in L^{2}\left(\mathbb{R}^{2}\right)$ and almost every $x \in \mathbb{R}^{2}$, with unconditional convergence in $L^{2}\left(\mathbb{R}^{2}\right)$. Applying a Fourier transform to this equation, we get

$$
\rho \hat{f}(\xi)=\sum_{k \in \mathcal{K}} \sum_{\gamma \in \Gamma_{k}}\left\langle f, \psi_{k}(\cdot-\gamma)\right\rangle e^{-i \gamma \cdot \xi} \widehat{\psi_{k}}(\xi),
$$

for almost every $\xi \in \mathbb{R}^{2}$, with

$$
\left\langle f, \psi_{k}(\cdot-\gamma)\right\rangle=\mathcal{F}\left(\hat{f} \widehat{\widehat{\psi_{k}}}\right)(-\gamma),
$$

where $\mathcal{F}$ denotes the Fourier transform. Hence

$$
\rho \hat{f}(\xi)=(2 \pi)^{-2} \sum_{k \in \mathcal{K}} \sum_{\gamma \in \Gamma_{k}} \mathcal{F}\left(\hat{f} \overline{\psi_{k}}\right)(-\gamma) e^{-i \gamma \cdot \xi} \widehat{\psi_{k}}(\xi) .
$$

Using Poisson summation formula

$$
\sum_{\gamma \in \Gamma_{k}} \hat{g}(\gamma) e^{i \gamma \cdot \xi}=\left|C_{\Gamma_{k}}\right| \sum_{\gamma \in \Gamma_{k}^{*}} g(\xi-\gamma),
$$


we obtain, let us say for all $f \in \mathcal{S}\left(\mathbb{R}^{2}\right)$,

$$
\rho \hat{f}(\xi)=(2 \pi)^{-2} \sum_{k \in \mathcal{K}}\left|C_{\Gamma_{k}}\right| \sum_{\gamma \in \Gamma_{k}^{*}} \hat{f}(\xi-\gamma) \overline{\psi_{k}(\xi-\gamma)} \widehat{\psi_{k}}(\xi) .
$$

Notice that

$$
\begin{aligned}
& \sum_{k \in \mathcal{K}}\left|C_{\Gamma_{k}}\right| \sum_{\gamma \in \Gamma_{k}^{*}}|\hat{f}(\xi-\gamma)|\left|\widehat{\psi_{k}}(\xi-\gamma)\right|\left|\widehat{\psi_{k}}(\xi)\right| \\
\leq & \sum_{\gamma \in \Gamma_{k}^{*}}|\hat{f}(\xi-\gamma)|\left(\sum_{k \in \mathcal{K}}\left|C_{\Gamma_{k}}\right|\left|\widehat{\psi_{k}}(\xi-\gamma)\right|^{2}\right)^{1 / 2}\left(\sum_{k \in \mathcal{K}}\left|C_{\Gamma_{k}}\right|\left|\widehat{\psi_{k}}(\xi)\right|^{2}\right)^{1 / 2} \\
\leq & C<\infty
\end{aligned}
$$

Then, by Fubini's Theorem,

$$
\rho \hat{f}(\xi)=(2 \pi)^{-2} \sum_{\gamma \in \bigcup_{k \in \mathcal{K}} \Gamma_{k}^{*}} \hat{f}(\xi-\gamma) \sum_{k \in \mathcal{K}_{\gamma}}\left|C_{\Gamma_{k}}\right| \overline{\widehat{\psi_{k}}(\xi-\gamma)} \widehat{\psi_{k}}(\xi) .
$$

Since $\min \left\{|x-y|: x, y \in \bigcup_{k \in \mathcal{K}} \Gamma_{k}^{*} \& x \neq y\right\}>0,(7)$ is satisfied, for all $f \in \mathcal{S}\left(\mathbb{R}^{2}\right)$, if and only if Conditions $a$ and $b$ are satisfied.

Conversely, assume that Conditions $a$ and $b$ are satisfied. Equation (7) is therefore satisfied for all $f \in \mathcal{S}\left(\mathbb{R}^{2}\right)$. The same arguments as above lead to (6). Now, suppose that $f \in L^{2}\left(\mathbb{R}^{2}\right)$. By the density of $\mathcal{S}\left(\mathbb{R}^{2}\right)$ in $L^{2}\left(\mathbb{R}^{2}\right)$, there is a sequence $\left\{f_{n}\right\}_{n}$ of functions in $\mathcal{S}\left(\mathbb{R}^{2}\right)$ that tends to $f$. Every $f_{n}$ satisfies (6) and consequently

$$
\rho\left\|f_{n}\right\|_{2}^{2}=\sum_{k \in \mathcal{K}} \sum_{\gamma \in \Gamma_{k}}\left|\left\langle f_{n}, \psi_{k}(\cdot-\gamma)\right\rangle\right|^{2} .
$$

By Fatou's Lemma, we know therefore that $\sum_{k \in \mathcal{K}} \sum_{\gamma \in \Gamma_{k}}\left|\left\langle f, \psi_{k}(\cdot-\gamma)\right\rangle\right|^{2}$ converges. It is then easy to prove that

$$
\rho\|f\|_{2}^{2}=\sum_{k \in \mathcal{K}} \sum_{\gamma \in \Gamma_{k}}\left|\left\langle f, \psi_{k}(\cdot-\gamma)\right\rangle\right|^{2},
$$

for all $f \in L^{2}\left(\mathbb{R}^{2}\right)$, by making $f_{n}$ tends to $f$ in (8). Hence the conclusion.

Below, we will need only the following sufficient conditions for a tight frame.

Corollary 1 Suppose $\left\|\psi_{k}\right\|_{2}=1, \forall k \in \mathcal{K}$ and

a. $\sum_{k \in \mathcal{K}_{\gamma}}\left|C_{\Gamma_{k}}\right|\left|\widehat{\psi_{k}}(\xi)\right|^{2}=(2 \pi)^{2} \rho$,

b. supp $\widehat{\psi_{k}}(\cdot-\gamma) \cap \operatorname{supp} \widehat{\psi_{k}}=\emptyset, \quad \forall k \in \mathcal{K}, \forall \gamma \in \Gamma_{k}$,

then $\left\{\psi_{k}(\cdot-\gamma)\right\}_{k \in \mathcal{K}, \gamma \in \Gamma_{k}}$ is a tight frame for $L^{2}\left(\mathbb{R}^{2}\right)$ with redundancy factor $\rho$.

A consequence of this result is that, for all $\rho>1$, there is a directional wavelet tight frame

$$
\left\{\psi_{k}(\cdot-\gamma)\right\}_{k \in \mathcal{K}, \gamma \in \Gamma_{k}}
$$




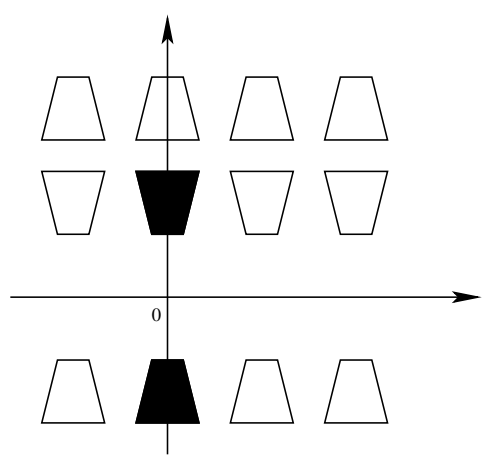

(a)

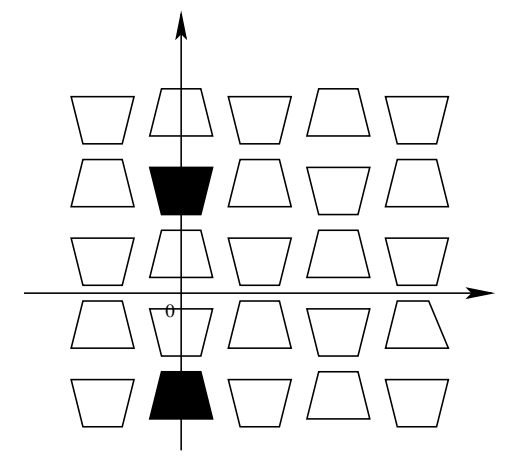

(b)

Figure 18: Packings $\{A+\{\gamma\}\}_{\gamma \in \Gamma}$ for (a) curvelets and (b) directional wavelets with dilation factor $<2$.

with redundancy factor $\rho$, and such that, for all $k \in \mathcal{K}, \psi_{k}$ has fast decay and $\widehat{\psi_{k}}$ is supported on a neighborhood of its ideal support. Her is an example that prove this claim. The idea is to construct a Shannon directional wavelet tight frame with arbitrary small redundancy factor $\rho>1$ using Proposition 3. If $d\left(A_{k}+\{\gamma\}, A_{k}+\right.$ $\{\eta\})>0$, for all $\gamma, \eta \in \Gamma_{k}^{*}$ with $\gamma \neq \eta$, and all $k \in \mathcal{K}$, where $d$ stands for the distance, then the wavelets $\widehat{\psi_{k}}$ are obtained by smoothing $\chi_{A_{k}}$ in such a way that, supp $\widehat{\psi_{k}}(\cdot-\gamma)$ and supp $\widehat{\psi_{k}}(\cdot-\eta)$ are disjoint for all $\gamma, \eta$ and $k$. Condition $a$ of Corollary 1 can also easily be satisfied in order to construct the sought regular tight frame.

The set $A=\left\{\xi \in \mathbb{R}^{2}: \quad \alpha\left|\xi_{2}\right|<\left|\xi_{1}\right|\right.$ and $\left.1<\left|\xi_{2}\right|<2\right\}$ considered in Section 2 cannot be used any longer. Indeed, if it is shifted along the same lattice as in Figure 3 -(a), the smoothed Fourier transforms of the wavelets cannot have disjoint supports. There are, on the other hand, lattices $\Gamma^{*}$ such that $\{A+\{\gamma\}\}_{\gamma \in \Gamma^{*}}$ is a packing of $\mathbb{R}^{2}$ and $d\left(A_{k}+\gamma, A_{k}+\eta\right)>0$ if $\gamma \neq \eta$, but their density cannot be arbitrary close to 1 . An example of such a packing is given in Figure 18-(a). (This kind of packing is used in the construction of curvelets[3].)

Consider henceforth the set

$$
A=\left\{\xi \in \mathbb{R}^{2}:\left|\xi_{1}\right|<\alpha\left|\xi_{2}\right| \text { and } 1<\left|\xi_{2}\right|<\beta\right\}
$$

with $\beta \in(1,2)$. When it is shifted along $\Gamma^{*}=\mathbb{Z} e_{1}+\mathbb{Z} e_{2}$ where $e_{1}=(0,(\beta+2) / 2)$ and $e_{2}=(\alpha(\beta+1)-\beta+2,(\beta+2) / 4)$, it forms a packing which density tends to 1 as $\beta$ tends to 2 . This example is shown in Figure 18-(b). We show in Figure 19-b, the frequency partitioning corresponding to the obtained $\beta$-adic directional wavelets.

Notice that in the limit case when $\beta=2$, we find again the tiling of Figure 3(a). It is, of course, not realistic to choose $\beta$ too close to 2 as, in such a case, the wavelets have no aliasing and fast decay but, by Heisenberg principle, their essential spatial support is too large (and the wavelets are therefore not sufficiently localized in the spatial domain). Moreover the discretization process needed for an implementation on images with a finite number of pixels, does not permit to make $\beta$ tends to 2 either. Nevertheless, we can expect to obtain properly localized (in space and frequency) wavelets tight frames with redundancy factor less than 2 while curvelets have a redundancy factor equal to $7.2[3]$. 


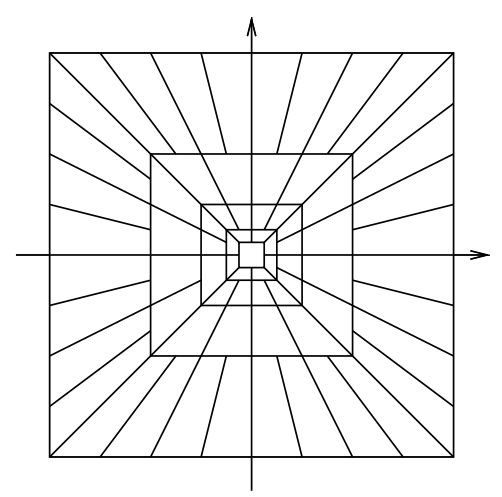

(a)

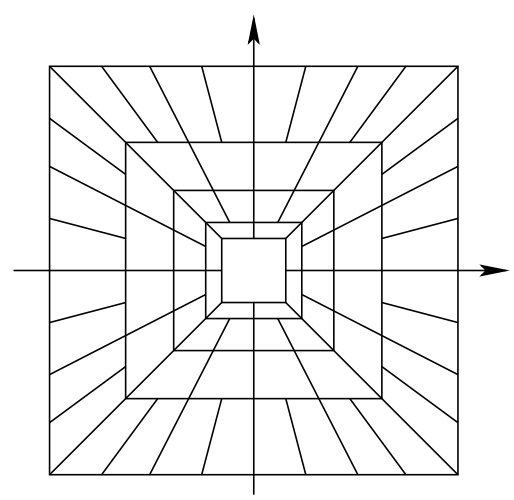

(b)

Figure 19: Packings $\left\{A_{k}\right\}_{k \in \mathcal{K}}$ for (a) curvelets and (b) directional wavelets with dilation factor $<2$.

\section{Conclusion and outlook}

The new directional wavelet transform that we proposed in Section 3 is implemented in a simple and fast algorithm. In some sense, it improves the curvelet and contourlet transforms as it is non redundant. However it has lost in frequency localization since the directional wavelets are aliased. In spite of this drawback, we have obtained encouraging numerical results. The later could be improved by developing new filter design techniques. In particular, FIR filters could be used when the transform is applied to hexagonally sampled images, and would permit to reduce the Gibbs and aliasing phenomenon.

Proposition 2 shows that we cannot construct directional wavelets (as they are defined in this article) without aliasing or redundancy. The proposed transform can therefore be very attractive when we cannot manage with redundant transforms as, for instance, in lossless compression. On the other hand, we shown in Section 5 that the redundancy factor of well localized directional wavelet tight frames can be dramatically reduced, and consequently that these wavelets could be used in low bit rate compression.

The discussion above leaves however some open questions. In particular, we shown that there are no biorthogonal directional wavelet bases that are both well localized in the sense that the wavelets and the dual wavelets have fast decay and their Fourier transform are supported on an arbitrary close neighborhood of their ideal support. But we do not know whether there are well localized directional wavelet bases whose dual basis is aliased. These transforms would be very useful since images could be reconstructed without aliasing artifact by reserving the well localized wavelet bases for the synthesis.

For instance, consider a Shannon directional wavelet basis given by the sets $\left\{A_{k}\right\}_{k \in \mathcal{K}}$ and the lattices $\left\{\Gamma_{k}\right\}_{k \in \mathcal{K}}$, with $A_{k}=D_{k}^{*} A$ and $\Gamma_{k}=D_{k}^{-1} \Gamma$ except possibly for one index $k$ corresponding to the low frequency wavelet $\phi$. Define $\psi$ by its Fourier transform

$$
\widehat{\psi}(\xi)=2 \pi|A|^{-1 / 2} \sqrt{g * \chi_{A}(\xi)} e^{i \alpha \cdot \xi}, \quad \forall \xi \in \mathbb{R}^{2},
$$

where $g$ is supported on a neighborhood of $0, \int_{\mathbb{R}^{2}} g(x) d x=1$ and $\alpha \notin \Gamma$ so that the 
wavelets are not too much aligned. It satisfies the identity

$$
\sum_{\gamma \in \Gamma^{*}}|\widehat{\psi}(\cdot-\gamma)|^{2}=\frac{(2 \pi)^{2}}{|A|}
$$

which means that $\{\psi(\cdot-\gamma)\}_{\gamma \in \Gamma}$ is an orthonormal basis for $\mathcal{W}=\overline{\langle\psi(\cdot-\gamma)\rangle_{\gamma \in \Gamma}}$. More generally, if $\psi_{k}=\psi \circ D_{k}$, then $\left\{\psi_{k}(\cdot-\gamma)\right\}_{\gamma \in \Gamma_{k}}$ is an orthonormal basis for $\mathcal{W}_{k}=\overline{\left\langle\psi_{k}(\cdot-\gamma)\right\rangle_{\gamma \in \Gamma_{k}}}$, but we do not know whether $\left\{\psi_{k}(\cdot-\gamma)\right\}_{k \in \mathcal{K}, \gamma \in \Gamma_{k}}$ is a basis for $L^{2}\left(\mathbb{R}^{2}\right)$. In particular, do we have

$$
L^{2}\left(\mathbb{R}^{2}\right)=\bigoplus_{k \in \mathcal{K}} \mathcal{W}_{k} ?
$$

\section{Appendix: Fast Fourier Transform on regular lat- tice.}

For convenience, we give a brief description of a fast algorithm for the computation of the Fourier transform of an image $I$ defined on a regular quotient lattice $\Gamma / \Lambda$ with $\Gamma \subset \Lambda \subset \mathbb{R}^{2}$.

The Fourier transform of $I$ is defined by

$$
\widehat{I}[\lambda]=\sum_{\gamma \in \Gamma / \Lambda} I[\gamma] e^{-i\langle\gamma, \lambda\rangle}, \quad \forall \lambda \in \Gamma^{*} / \Lambda^{*} .
$$

Notice that the inverse Fourier transform is given by the reconstruction formula

$$
I[\gamma]=\frac{1}{\#(\Gamma / \Lambda)} \sum_{\lambda \in \Lambda^{*} / \Gamma^{*}} \widehat{I}[\lambda] e^{i\langle\gamma, \lambda\rangle}, \quad \forall \gamma \in \Lambda / \Gamma,
$$

so that the FFT and the IFFT can be computed using a similar scheme. Let us show that they are implemented in a $O(N \log N)$ algorithm where $N=\#(\Gamma / \Lambda)$. Suppose first that $2 \Gamma \subset \Lambda$ and write $\Gamma$ as

$$
\Gamma=\bigcup_{i=0}^{3}\left(2 \Gamma+\left\{\gamma_{i}\right\}\right)
$$

with $\gamma_{i} \in \Gamma, \forall i \in\{0, \cdots, 3\}$ (and $\gamma_{0}=0$ ). Thus we have

$$
\begin{aligned}
\widehat{I}[\lambda] & =\sum_{i=0}^{3} \sum_{\gamma \in \Gamma /\left(2^{-1} \Lambda\right)} I\left[2 \gamma+\gamma_{i}\right] e^{-i\langle\gamma, 2 \lambda\rangle} e^{-i\left\langle\gamma_{i}, \lambda\right\rangle} \\
& =\sum_{i=0}^{3} \widehat{I}_{i}[2 \lambda] e^{-i\left\langle\gamma_{i}, \lambda\right\rangle}
\end{aligned}
$$

where $I_{i}=\left(\gamma \mapsto I\left[2 \gamma+\gamma_{i}\right]\right)$ is defined on $\Gamma /\left(2^{-1} \Lambda\right)$ with $\#\left(\Gamma /\left(2^{-1} \Lambda\right)\right)=\#(\Gamma / \Lambda) / 4$. So, to compute $\widehat{I}$, one just need to compute the Fourier transform of the 4 smaller images $I_{i}$ and multiply them by $e^{-i\left\langle\gamma_{i}, \lambda\right\rangle}$.

Suppose that $\#(\Gamma / \Lambda)=4^{n}$ and denote by $C_{n}$ the computation cost of $\widehat{I}$. If one neglects the cost of sums, we obtain the recurrence relation

$$
C_{n}=4 C_{n-1}+4^{n} .
$$


(Since $\gamma_{0}=0$, we have actually $C_{n}=4 C_{n-1}+34^{n-1}$.) We can decrease $n$ until $2 \Gamma \not \subset \Lambda$. In such a case, we find another lattice $\Delta \subset \Lambda$ such that

$$
\Gamma=\bigcup_{i=0}^{3}\left(\Delta+\left\{\gamma_{i}\right\}\right),
$$

and apply the same process. As $C_{0}=0$, we get $C_{n}=4^{n} n$. As a conclusion, for an image of size $N=4^{n}$, the computation cost is $N \log _{4} N$.

\section{References}

[1] J-P. Antoine and R. Murenzi, Two-dimensional directional wavelets and the scale-angle representation, Signal Processing 52: 259-281, 1996.

[2] R. H. Bamberger and M. J. T. Smith, A filter bank for the directional decomposition of images: theory and design, IEEE Trans. Signal Proc., 40(4): 882-893, 1992.

[3] E. J. Candès, L. Demanet, D. L. Donoho and L. Ying, Fast Dicrete Curvelet Transform, SIAM Multiscale Model. Simul. 5(3): 861-899, 2006.

[4] E. J. Candès and D. L. Donoho, Ridgelets - a key to higher dimensional intermittency?, Phil. Trans. R. Soc. Lond. A., pp:2495-2509, 1999.

[5] E. J. Candès and D. L. Donoho, Curvelets - a suprizingly effective nonadaptive representation for objects with edges, Curve and Surface Fitting, Vanderbuilt Univ. Press, 1999.

[6] E. J. Candès and D. L. Donoho, Curvelets - Multiresolution representation and scaling laws, SPIE Wavelet Applications in Signal and Image Processing VIII, Vol. 4119, 2000.

[7] T. Chen and P. Vaidyanathan, Considerations in multidimensional filter bank design, in Proc. of IEEE Int. Symp. on Circuits and Systems, 643-645, 1993.

[8] A. Cohen, I. Daubechies and J. C. Feauveau, Biorthogonal bases of compactly supported wavelets, Comm. in Pure and Appl. Math., 45(5):485-560, 1992.

[9] A. Cohen and J. M. Schlenker, Compactly supported bidimensional wavelet bases with hexagonal symmetry, Constructive Approximation, 9:209-236, 1993.

[10] A. L. Cunha and M. N. Do, On the two-channel filter banks with directional vanishing moments, IEEE Trans. on Image Processing, 16(5): 1207-1219, 2007.

[11] M. N. Do and M. Vetterli, Contourlets, Beyond Wavelets, Academic Press, New York, 2003.

[12] D. L. Donoho, Orthonormal ridgelets and linear singularities, SIAM Journal on Math. Analysis, 31(5):1062-1099, 2000.

[13] S. Durand, M-band filtering and non-redundant directional wavelets, Applied and Comput. Harmonic Analysis, 22(1):124-139, 2007.

[14] K. Guo, D. Labate, W. Q. Lim, G. Weiss and E. Wilson, Wavelets with composite dilations and their MRA properties, Applied and Comput. Harmonic Analysis, 20: 202-236, 2006.

[15] B. Grünbaum and G. C. Shephard, Tilings and paterns: an introduction, Freeman \& Co., 1989.

[16] N. G. Kingsbury, The dual-tree complex wavelet transform: A new technique for shift invariance and directional filters, in proc. IEEE DSP, 1998.

[17] E. Le Pennec and S. Mallat, "Sparse geometrical image representation with bandelets", IEEE Trans. on Image Processing, 14(4): 423-439, 2005.

[18] S. Mallat, A Wavelet Tour of Signal Processing, Academic Press, London, 1999.

[19] R. M. Mersereau, The processing of hexagonally sampled two-dimensional signal, Proc. IEEE 67(6): 930-949, 1979.

[20] F. G. Meyer and R. R. Coifman, "Brushlets: a tool for directional image analysis and image compression", Applied and Comput. Harmonic Analysis, 5: 147-187, 1997.

[21] R. Murenzi, Ondelettes multidimensionnelles et applications à l'analyse d'images, PhD Thesis, Univ. Cath. Louvain, Belgium, 1990.

[22] T. T. Nguyen and S. Oraintara, Multiresolution Direction Filter Banks: Theory, Design and Applications, IEEE Trans. Signal Proc., 3895-3905, 2005. 
[23] I. W. Selesnick, R. G. Baraniuk and N. Kingsbury, The dual-tree complex wavelet transform - A coherent framework for multiscale signal and image processing, IEEE Trans. Signal Proc., 22(6): 123-151, 2005.

[24] E. P. Simoncelli and E. H. Adelson, Subband Image Coding with Hexagonal Quadrature Mirror Filters, Picture Coding Symposium, Cambridge, MA, 1990

[25] E. P. Simoncelli, W. T. Freeman, E. H. Adelson and D. J. Heeger, Shiftable Multiscale Transforms, IEEE Trans. Info. Theory, 38(2): 587-607, 1992

[26] D. B. H. Tay and N. G. Kingsbury, Flexible design of multidimensional perfect reconstruction FIR 2-band filters using transformations of variables, IEEE Trans. Image Proc. 2:466-480, 1993. 